\title{
Exponential Dynamical Anderson Localization in N-particle Models on Graphs with Infinite-range Interaction
}

\author{
Victor Chulaevsky \\ Department of Mathematics, University of Reims Champagne-Ardenne, France
}

Copyright (C) 2015 by authors, all rights reserved. Authors agree that this article remains permanently

open access under the terms of the Creative Commons Attribution License 4.0 International License

\begin{abstract}
We extend the techniques and results of the multi-particle variant of the Fractional Moment Method, developed by Aizenman and Warzel, to disordered quantum systems in general finite or countable graphs with polynomial growth of balls, in presence of an exponentially decaying interaction. In the strong disorder regime, we prove complete exponential multi-particle strong localization. Prior results, obtained with the help of the multi-scale analysis, proved only a sub-exponential decay of eigenfunction correlators.
\end{abstract}

Keywords Multi-particle Anderson Localization, Eigen-value Concentration Estimates

\section{Introduction}

\section{Introduction: The motivation and the model}

The rigorous multi-particle Anderson localization theory is a relatively recent direction in the spectral theory of disordered media. The first results in this direction, establishing the stability of Anderson localization in a two-particle system in $\mathbb{Z}^{d}$ with respect to a short-range interaction [16], have been immediately followed by the proofs of exponential spectral localization (cf. $[5,17]$ ) and exponential strong dynamical localization (cf. [5]) in $N$-particle systems, for any fixed $N \geq 2$.

In the multi-particle models with finite-range interaction, the MPFMM, when applicable, provides the strongest decay bounds upon the eigenfunction correlators (EFC), as does its original, single-particle variant. In particular, such bounds are stronger than those proved with the help of the multiparticle MSA (MPMSA), provided both methods apply to the same model. However, the relations between the two approaches are more complex in the realm of multi-particle, interactive models than for the systems with no interaction. In particular, in the situation where the interaction potential decays slower than exponentially, the existing techniques (based on the MPFMM or the MPMSA) allow one to prove only a sub-exponential decay of the EFCs; in the case of the MPFMM this results in a similar - sub-exponential - decay of the eigenfunctions, for the latter is derived from the analysis of the fractional moments and, ultimately, of the eigenfunction correlators. The MPMSA is free from this limitation, since one can carry out independent analyses of the EFC and of the EFs. Exponential decay of the EFs in presence of a sub-exponentially decaying interaction was proved in discrete $N$-particle Anderson models (cf. [19]) and in a class of continuous models with the so-called alloy-type random potential (cf. [11]).

For these reasons, we consider in the present paper only the case of exponentially decaying interactions, where an extension of the MPFMM techniques developed by Aizenman and Warzel [5] allows us to prove exponential decay of the eigenfunctions and of their correlators.

\subsection{The multi-particle Hamiltonian}

Consider a finite or countable connected graph $(\mathcal{Z}, \mathcal{E})$ without cyclic edges endowed with the canonical graph distance $\mathrm{d}=\mathrm{d}^{(\mathcal{Z})}: \mathrm{d}(x, y)$ is the length of the shortest path from $x$ to $y$ over the graph edges; $\mathrm{d}(x, x)=0$. We assume that

$$
\forall L \geq 1 \quad \sup _{x \in \mathcal{Z}} \operatorname{card} \mathrm{B}_{L}(x) \leq C_{d} L^{d} .
$$

Further, consider the Cartesian power $\mathcal{Z}^{N}, N \geq 2$, with the graph structure defined as follows: $\mathbf{x}=\left(x_{1}, \ldots, x_{N}\right)$ and $\mathbf{y}=\left(y_{1}, \ldots, y_{N}\right)$ form an edge iff there exists $j$ such that $\mathrm{d}\left(x_{j}, y_{j}\right)=1$ and $x_{i}=y_{i}$ for all $i \in\{1, \ldots, N\} \backslash\{j\}$. The graph with the vertex set $\mathcal{Z}^{N}$ and the defined edge set will be denoted by $\mathcal{Z}^{N}$. For $\mathcal{Z}=\mathbb{Z}^{d}$, this construction gives rise to the graph $\left(\mathbb{Z}^{d}\right)^{N} \cong \mathbb{Z}^{N d}$ with the usual graph structure.

The vertices of $\mathcal{Z}^{N}$ represent the configurations of $N$ distinguishable quantum particles in $\mathcal{Z}$. An important characteristics of $\mathbf{x}=\left(x_{1}, \ldots, x_{N}\right)$ is its support $\Pi \mathbf{x}:=\left\{x_{1}, \ldots, x_{N}\right\}$. (Notice that in a system of $N$ indistinguishable Fermiparticles, $\Pi \mathrm{x}$ is the configuration.) We define the diameter of a configuration by

$$
\operatorname{diam} \mathbf{x} \equiv \operatorname{diam} \Pi \mathbf{x}=\max _{y, z \in \Pi \mathbf{x}} \mathrm{d}(y, z)
$$


It will be convenient to use also the max-distance in $\mathcal{Z}^{N}$,

$$
\boldsymbol{\rho}(\mathbf{x}, \mathbf{y})=\max _{1 \leq i \leq N} \mathrm{~d}\left(x_{i}, y_{i}\right)
$$

and the so-called Hausdorff (pseudo-)-distance, defined actually for the supports $\Pi \mathbf{x}, \Pi \mathbf{y}$ and formally extended to the configurations:

$$
\mathrm{d}_{\mathcal{H}}(\mathbf{x}, \mathbf{y})=\max \left[\max _{x \in \Pi \mathbf{x}} \mathrm{d}(x, \Pi \mathbf{y}), \max _{y \in \Pi \mathbf{y}} \mathrm{d}(y, \Pi \mathbf{x})\right] .
$$

A more natural object than the max-distance is its symmetrized counterpart

$$
\mathrm{d}_{\mathrm{S}}(\mathbf{x}, \mathbf{y})=\min _{\pi \in \mathfrak{S}_{N}} \boldsymbol{\rho}(\mathbf{x}, \pi(\mathbf{y}))
$$

here the elements $\pi$ of the symmetric group $\mathfrak{S}_{N}$ act on configurations by permutations of the particle positions. As we explain below, the localization bounds in our model can be established only in terms of a permutation-invariant metric in the $N$-particle configuration space. Note that $\mathrm{d}_{\mathcal{H}}$ is also permutation-invariant. An important relation between $d_{S}$ and $\mathrm{d}_{\mathcal{H}}$ is the following inequality:

$$
\mathrm{d}_{\mathcal{H}}(\mathbf{x}, \mathbf{y}) \geq \mathrm{d}_{\mathrm{S}}(\mathbf{x}, \mathbf{y})-\min [\operatorname{diam} \mathbf{x}, \operatorname{diam} \mathbf{y}] .
$$

We study a random bounded self-adjoint operator in $\ell^{2}\left(\mathcal{Z}^{N}\right)$, of the form

$$
\mathbf{H}(\omega)=\sum_{j=1}^{N}\left(-\Delta^{(j)}+g V\left(x_{j} ; \omega\right)\right)+\mathbf{U}(\mathbf{x}),
$$

where $V: \mathcal{Z} \times \Omega \rightarrow \mathbb{R}$ is a random field on $\mathcal{Z}$ (the singleparticle configuration space), relative to some probability space $(\Omega, \mathfrak{F}, \mathbb{P}), \mathbf{U}$ is the operator of multiplication by the function

$$
\mathbf{x}=\left(x_{1}, \ldots, x_{N}\right) \mapsto \sum_{i \neq j} U\left(\left|x_{i}-x_{J}\right|\right),
$$

generated by a two-body interaction potential $\mathbb{N} \ni r \mapsto U(r)$, and $\Delta^{(j)}$ are replicas of the canonical graph Laplacian on $\mathcal{Z}$, acting on the respective variables (particle positions) $x_{j}$.

\subsection{Assumptions}

We assume that the interaction potential satisfies the following condition.

$$
\text { (U1) } \forall r \geq 0 \quad|U(r)| \leq C \mathrm{e}^{-a r}, \quad C, a \in(0,+\infty) .
$$

Our main assumption on the external (random) potential is as follows.

(V1) The random field $V: \mathcal{Z} \times \Omega \rightarrow \mathbb{R}$ is IID, a.s. bounded, with

$$
\mathbb{P}\{V(x ; \omega) \in[0,1]\}=1,
$$

and admits a bounded marginal probability density $p_{V}(\cdot)$.

The assumption of positivity is not essential, for a bounded random potential $V$, since one can introduce a new potential $\widetilde{V}(x ; \omega):=V(x ; \omega)-\inf V \geq 0$, and this simply results in a global energy shift, leaving invariant all eigenfunctions. Restricting the support to the interval $[0,1]$ is convenient, but does not result in a loss of generality, due to the presence of the amplitude $g$ in the random potential $g V(\cdot ; \omega)$.

\subsection{Main results}

Theorem 2.1. Assume that the interaction potential $U$ in the Hamiltonian $\mathbf{H}(\omega)$ decays exponentially fast at infinity (cf. Assumption (U2)) and the external random potential satisfies Assumption (V1). For $g_{0}$ large enough and for all $g$ with $|g| \geq g_{0}, \mathbf{H}(\omega)$ exhibits exponential decay of the fractional moments of the Green functions. Specifically, let $I \subset \mathbb{R}$ be an interval of length $|I|<\infty$, then there exists some $m=m(g)>0$ such that for any pair of $N$-particle configurations $\mathbf{x}, \mathbf{y}$ one has

$$
\int_{\mathbb{R}} \mathbb{E}\left[|\mathbf{G}(\mathbf{x}, \mathbf{y} ; E)|^{s}\right] d E \leq|I| \mathrm{e}^{-m \mathrm{~d}_{\mathcal{H}}(\mathbf{x}, \mathbf{y})} .
$$

Remark 2.1. $m(g) \rightarrow+\infty$ as $|g| \rightarrow+\infty$.

Theorem 2.2. Consider an N-particle Anderson model with the single-particle configuration space $\mathbb{Z}^{1}$ and the Hamiltonian

$$
\mathbf{H}_{h}(\omega)=-\boldsymbol{\Delta}+g \mathbf{V}(\mathbf{x} ; \omega)+h \mathbf{U}(\mathbf{x}), \quad h \in \mathbb{R} .
$$

Assume that the inter-particle interaction potential $U$ decays exponentially fast at infinity (cf. Assumption (U2)) and the external random potential satisfies Assumption (V1). For any $g \neq 0$ there exist $m>0$ and $h_{\circ}=h_{\circ}\left(|g|, F_{V},\|\mathbf{U}\|\right)>0$ such that for all $h \in\left[-h_{\circ}, h_{\circ}\right]$, the random Hamiltonian $\mathbf{H}_{h}(\omega)$ exhibits exponential decay of the fractional moments of the Green functions: for any bounded interval $I \subset \mathbb{R}$,

$$
\int_{I} \mathbb{E}\left[|\mathbf{G}(\mathbf{x}, \mathbf{y} ; E)|^{s}\right] d E \leq|I| \mathrm{e}^{-m \mathrm{~d}_{\mathcal{H}}(\mathbf{x}, \mathbf{y})} .
$$

Theorem 2.3. The exponential decay of the fractional moments of the form (2.6)-(2.7) implies complete exponential strong dynamical localization: for any configurations $\mathbf{x}, \mathbf{y}$

$$
\mathbb{E}\left[\sup _{t \in \mathbb{R}}\left\langle\mathbf{1}_{\mathbf{y}}\left|\mathrm{e}^{-\mathrm{i} t \mathbf{H}(\omega)}\right| \mathbf{1}_{\mathbf{x}}\right] \leq C(\mathbf{x}) \mathrm{e}^{-m^{\prime} \mathrm{d}_{\mathrm{S}}(\mathbf{x}, \mathbf{y})} .\right.
$$

Our proofs are closer to the original technique from [5] than to a more advanced method developed by Fauser and Warzel [21].

\section{Basic notation and preliminary re- marks}

We will systematically make use of the elementary inequality which is one of the cornerstones of the FMM technique: $\forall s \in(0,1)\left|\sum_{n} a_{n}\right|^{s} \leq \sum_{n}\left|a_{n}\right|^{s}$.

Another standard ingredient of the localization analysis is the second resolvent identity, $(A+B)^{-1}=A^{-1}-A^{-1} B(A+$ $B)^{-1}=A^{-1}-(A+B)^{-1} B A^{-1}$, valid as long as $A$ and $(A+B)$ are invertible elements of some algebra. It will be always used in a situation where $A$ and $B$ are linear operators in finite-dimensional (Hilbert) spaces, so there is no need to address the issue of boundedness and domains. Often, albeit not always, it will be used in the case where $A=A_{1} \oplus A_{2}: \mathcal{H} \rightarrow \mathcal{H}$,

$$
\mathcal{H}=\ell^{2}\left(\Lambda_{1}\right) \oplus \ell^{2}\left(\Lambda_{2}\right) \cong \ell^{2}\left(\Lambda_{1} \sqcup \Lambda_{2}\right), \quad A_{j}: \ell^{2}\left(\Lambda_{j}\right) \rightarrow \ell^{2}\left(\Lambda_{j}\right),
$$

$\Lambda_{j} \subset \mathcal{Z}$ being finite subsets with the boundaries $\mathcal{D}_{j}=\partial^{-} \Lambda_{j}$, $\mathcal{D}_{1} \cap \mathcal{D}_{2}=: \mathcal{D} \neq \varnothing$. As to the operator $B$, it has the form (in Dirac's "ket-bra" notation)

$$
\sum_{\langle x y\rangle}\left(\left|\mathbf{1}_{x}\right\rangle\left\langle\mathbf{1}_{y}|+| \mathbf{1}_{y}\right\rangle\left\langle\mathbf{1}_{x}\right|\right),
$$


where $\langle x y\rangle$ runs over all pairs with $x \in \mathcal{D}_{1}, y \in \mathcal{D}_{2}$ and $\mathrm{d}(x, y)=1$. In this case the resolvent equation takes the form often called the Geometric Resolvent Equation (GRE) and implies the Geometric Resolvent Inequality (GRI), sometimes also referred to as the Simon-Lieb Inequality (SLI). Its FMM-flavoured variant (which will be referred to as the FGRI = Fractional GRI) reads as follows:

$$
\left|G_{\Lambda}(x, y ; E)\right|^{s} \leq \sum_{\left\langle w, w^{\prime}\right\rangle \in \mathcal{D}_{1} \times \mathcal{D}_{2}}\left|G_{\Lambda_{1}}(x, w ; E)\right|^{s}\left|G_{\Lambda}\left(w^{\prime}, y ; E\right)\right|^{s} .
$$

Following [5], we introduce the energy-disorder expectation $\widehat{\mathbb{E}}^{I}[\cdot]$ : given an interval $I \subset \mathbb{R}$ of length $|I| \geq 1$ and a measurable function $f: \mathbb{R} \times \Omega$, we set

$$
\widehat{\mathbb{E}}^{I}[f(E, \omega)]:=|I|^{-1} \int_{I} \mathbb{E}[f(E, \omega)] d E,
$$

where $\mathbb{E}[\cdot]$ is the conventional expectation relative to $(\Omega, \mathbb{P})$. The benefits of averaging over the augmented, energydisorder probability space are two-fold:

1. In the fractional moment analysis of the Green functions, it allows one to focus on the decay properties of the eigenfunction correlators and prove a crucial result on finiteness of the fractional moments by a "soft" argument, based on the Boole identity (cf. Appendix C) and an explicit integration in the energy.

2. The derivation of the decay bounds on the eigenfunction correlators from the bounds on the fractional moments of the Green functions, used in our paper (cf. Sect 3.1), employs a Chebyshev-type argument in the energy-disorder space rather than in the disorder space, so the bounds in terms of the expectations $\widehat{\mathbb{E}}^{I}[\cdot]$ turn out to be a natural tool for such a derivation.

The following elementary statement explains what makes the 2-particle systems special in the framework of our analysis:

$$
\forall \mathbf{x}, \mathbf{y} \in \mathcal{Z}^{2} \quad \mathrm{~d}_{H}(\mathbf{x}, \mathbf{y})=\mathrm{d}_{\mathrm{S}}(\mathbf{x}, \mathbf{y}) .
$$

For example, with $\mathcal{Z}=\mathbb{Z}^{d}$, the Hausdorff distance is the symmetrized version of the genuine norm-distance in $\left(\mathbb{Z}^{d}\right)^{2} \cong$ $\mathbb{Z}^{2 d}$. Thus the 2-particle decay estimates established in the Hausdorff distance imply those in the (symmetrized) normdistance.

\subsection{Finiteness of the fractional moments}

A priori bounds on the fractional moments of the resolvents have been one of the inescapable ingredients of the FMM since its inception in [1]. Their adaptation to the multiparticle models (cf. [5]) is, however, more involved than in the 1-particle theory. Both Ref. [5] and a more recent work [21], as well as [4] dedicated to the FMM for differential random operators, refer to some general results on maximally dissipative operators and related topics; cf., e.g., [4], [8, 33].

We will need the following statement.

Lemma 3.1. For any $s \in(0,1)$ there exists $C_{s}<\infty$ such that for any finite connected subset $\Lambda \subset \mathcal{Z}$, any two sites $u_{1}, u_{2} \in \mathcal{Z}$, with $n:=$ card $\left\{u_{1}, u_{2}\right\} \in\{1,2\}$, and any pair of configurations $\mathbf{x}, \mathbf{y} \in \Lambda^{2}$ with $\Pi \mathbf{x} \ni u_{1}$, Пy $\ni u_{2}$, the following bound holds:

$$
\mathbb{E}\left[|\mathbf{G}(\mathbf{x}, \mathbf{y} ; E)|^{s} \mid \mathfrak{F}_{\neq u_{1}, u_{2}}\right] \leq M_{s}<+\infty
$$

where

$$
M_{s}=M_{s}\left(s, g, F_{V}, n\right) \leq C\left(F_{V}, n\right)|g|^{-s},
$$

for some $C\left(F_{V}, n\right)<\infty$.

In Ref. [5], the proof of such an a priori bound has been clearly outlined, with direct references to prior works containing necessary analytic and probabilistic results and making the proof complete; it does not rely on any specific form of the interaction potential, be it of finite or infinite range, since only the random potential is used in the main argument. However, for the reader's convenience, we provide in Appendix D a detailed, self-contained proof of Lemma 3.1 relying on a bare minimum of analytic tools, including the Birman-Schwinger relation for finite-dimensional operators and the Boole formula [7] (proved in Appendix C; the remarkably short and elementary argument is due to Loomis [28]). The proof shows that $M_{s}$ is a continuous function of the interaction potential. In particular, for the interaction of the form $h \mathbf{U}(\mathbf{x})$, with fixed $\mathbf{U}(\cdot)$ and $h \in \mathbb{R}$, it depends continuously on $h$; this fact is used in the application of the MPFMM to the perturbations of the non-interacting $N$-particle system by weak interactions $h \mathbf{U},|h| \ll 1$.

\subsection{From the fractional moments to the EFCs}

This subsection may prove to be of limited interest to a reader familiar with modern methods of derivation of strong dynamical localization from the suitable estimates, in probability or in expectation, obtained through a fixed-energy localization analysis. The main goal here is to show that the scaling analysis performed in Section 3 implies indeed $N$ particle dynamical localization for any given $N>1$, provided the localization for the single-particle systems is sufficiently strong.

We employ an argument developed by Elgart et al. [20]; this is in essence an advanced version of an older Chebyshevtype argument given by Martinelli and Scoppola [29] in the general context of the MSA (the FMM was not known yet in 1985). Its form given below appeared in [9] and [18].

Given a ball $\mathbf{B}_{L}(\mathbf{z})$, introduce a function

$$
\begin{aligned}
(E, \omega) \mapsto \mathbf{F}_{\mathbf{z}}(E ; \omega) & \equiv \mathbf{F}_{\mathbf{z}, L}(E ; \omega) \\
& :=\max _{\mathbf{y} \in \partial^{-} \mathbf{B}_{L}(\mathbf{z})}\left|\mathbf{G}_{\mathbf{B}_{L}(\mathbf{z})}(\mathbf{z}, \mathbf{y} ; E ; \omega)\right| .
\end{aligned}
$$

In a number of formulae below, the radius $L$ will be fixed (and clear from the context), so it will be often omitted from notation $\mathbf{F}_{\mathbf{z}, L}$ for brevity.

We denote by $\Sigma\left(\mathbf{H}_{\mathbf{B}_{L}(\mathbf{z})}(\omega)\right)$ the (finite) spectrum of the operator $\mathbf{H}_{\mathbf{B}_{L}(\mathbf{z})}(\omega)$.

Lemma 3.2. Let be given a ball $\mathbf{B}_{L}(\mathbf{z})$, a bounded interval $I \subset \mathbb{R}$, and real numbers $a_{L}, b_{L}, c_{L}, q_{L}>0$ satisfying

$$
b_{L} \leq \min \left\{a_{L} c_{L}^{2}, c_{L}\right\}
$$

and

$$
\mathbb{P}\left\{\mathbf{F}_{\mathbf{z}}(E ; \omega)>a_{L}\right\} \leq q_{L} .
$$

Then there exists an event $\mathcal{S}_{b, \mathbf{z}}$ such that $\mathbb{P}\left\{\mathcal{S}_{b, \mathbf{z}}\right\} \leq b_{L}^{-1} q_{L}$ and for any $\omega \notin \mathcal{S}_{b, \mathbf{z}}$, the set of energies

$$
\left\{E \in I: \mathbf{F}_{\mathbf{z}}(E ; \omega)>2 a_{L}\right\}
$$

is covered by a union of intervals

$$
\bigcup_{i=1}^{K_{\mathbf{z}}} I_{j}, \quad I_{j}=\left\{E:\left|E-\lambda_{j}\right| \leq 2 c_{L}\right\}, \quad K_{z} \leq\left|\mathbf{B}_{L}(\mathbf{z})\right|,
$$


centered at the eigenvalues $\lambda_{j} \in \Sigma\left(\mathbf{H}_{\mathbf{B}_{L}(\mathbf{z})}(\omega)\right) \cap I$.

Proof. Consider the random Borel subsets of $I$ parameterized by $a^{\prime}>0$ (recall that $\mathbf{F}_{\mathbf{z}}$ is continuous outside the finite spectrum of $\left.\mathbf{H}_{\mathbf{B}_{L}(\mathbf{z})}\right)$

$$
\mathscr{E}_{a^{\prime}, \mathbf{z}}(\omega)=\left\{E \in I: \mathbf{F}_{\mathbf{z}}(E)>a^{\prime}\right\}
$$

and, with $a_{L}$ satisfying (3.3), the events parameterized by $b^{\prime}>$ 0 :

$$
\mathcal{S}_{b^{\prime}, \mathbf{z}}:=\left\{\omega: \operatorname{mes}\left(\mathscr{E}_{a_{L}, \mathbf{z}}\right)>b^{\prime}\right\} .
$$

Assuming (3.3)-(3.4), apply Chebyshev's inequality and the Fubini theorem:

$$
\begin{aligned}
\mathbb{P}\left\{\mathcal{S}_{b_{L}, \mathbf{z}}\right\} & \leq b_{L}^{-1} \mathbb{E}\left[\int_{I} \mathbf{1}_{\mathbf{F}_{\mathbf{z}}(E)>a_{L}} d E\right] \\
& =b_{L}^{-1} \int_{I} \mathbb{E}\left[\mathbf{1}_{\left.\mathbf{F}_{\mathbf{z}}(E)>a_{L}\right] d E}\right. \\
& =b_{L}^{-1} \int_{I} \mathbb{P}\left\{\mathbf{F}_{\mathbf{z}}(E)>a_{L}\right\} d E \leq b_{L}^{-1}|I| q_{L}
\end{aligned}
$$

Fix any $\omega \notin \mathcal{S}_{b_{L}, \mathbf{z}}$, so that mes $\left(\mathscr{E}_{\mathbf{Z}}\left(a_{L} ; \omega\right)\right) \leq b_{L}$, and consider the random sets parameterized by $c^{\prime}>0$ :

$$
\mathscr{R}\left(c^{\prime}\right):=\left\{\lambda \in \mathbb{R}: \min _{j}\left|\lambda_{j}(\omega)-\lambda\right| \geq c^{\prime}\right\} .
$$

Note that for $0<b_{L} \leq c_{L}$, we have

$$
\mathscr{A}_{b_{L}}:=\left\{E: \operatorname{dist}\left(E, \mathscr{R}\left(2 c_{L}\right)\right)<b_{L}\right\} \subset \mathscr{R}\left(c_{L}\right),
$$

hence the set $\mathscr{A}_{b_{L}}^{\mathrm{c}}$ is a union of sub-intervals at distance $\geq c_{L}$ from the spectrum. Let us show that

$$
\forall \omega \notin \mathcal{S}_{b_{L}, \mathbf{z}} \quad\left\{E: \mathbf{F}_{\mathbf{z}}(E ; \omega) \geq 2 a_{L}\right\} \cap \mathscr{R}\left(2 c_{L}\right)=\varnothing .
$$

Assume otherwise and pick any point $\lambda^{*}$ in the non-empty intersection figuring in the LHS. Let $J:=\left\{E^{\prime}:\left|E^{\prime}-\lambda^{*}\right|<\right.$ $\left.b_{L}\right\} \subset \mathscr{A}_{b_{L}} \subset \mathscr{R}\left(c_{L}\right)$, so for any $E \in J$ we have $\|\mathbf{G}(E)\| \leq c_{L}^{-1}$. Further, by the second resolvent identity, for any $E \in J$ we have

$$
\begin{aligned}
\|\mathbf{G}(E)\| & \geq\left\|\mathbf{G}\left(\lambda^{*}\right)\right\|-\left|\lambda^{*}-E\right|\left\|\mathbf{G}\left(\lambda^{*}\right)\right\|\|\mathbf{G}(E)\| \\
& \geq 2 a_{L}-b c_{L}^{-2} \geq a_{L},
\end{aligned}
$$

owing to (3.3). Therefore, $\mathscr{E}_{b} \supset J$, which is impossible, since mes $\mathscr{E}_{b_{L}, \mathbf{z}}(\omega) \leq b_{L}$ while mes $J=2 b_{L}$.

The obtained contradiction completes the proof.

Theorem 3.1. Let be given a bounded interval $I \subset \mathbb{R}$ and real numbers $a_{L}, b_{L}, c_{L}, q_{L}>0$ satisfying (3.3) and

$$
\max _{\mathbf{z} \in\{\mathbf{x}, \mathbf{y}\}} \sup _{E \in I} \mathbb{P}\left\{\mathbf{F}_{\mathbf{z}}(E)>a_{L}\right\} \leq q_{L} .
$$

Assume also that for some function $f:[0,1] \rightarrow \mathbb{R}_{+}$one has

$$
\mathbb{P}\left\{\operatorname{dist}\left(\Sigma\left(H_{\mathrm{B}_{L}(x)}\right), \Sigma\left(H_{\mathrm{B}_{L}(y)}\right)\right) \leq s\right\} \leq f(s) .
$$

Then

$$
\begin{array}{r}
\mathbb{P}\left\{\exists E \in \mathbb{R}: \min \left\{\mathbf{F}_{\mathbf{x}}(E), \mathbf{F}_{\mathbf{y}}(E)\right\}>a_{L}\right\} \\
\leq 2|I| b^{-1} q_{L}+f\left(4 c_{L}\right) .
\end{array}
$$

Proof. Introduce the events $\mathcal{S}_{b_{L}, \mathbf{z}}=\{\omega: \operatorname{mes}\{E \in I$ : $\left.\left.\mathbf{F}_{\mathbf{z}}(E)>a_{L}\right\}>b_{L}\right\}$, and the random sets $\mathscr{E}_{a_{L}, \mathbf{z}}(\omega):=\{E \in$ $\left.I: \mathbf{F}_{\mathbf{z}}(E ; \omega)>a_{L}\right\}$, for $\mathbf{z} \in\{\mathbf{x}, \mathbf{y}\}$, as in Lemma 3.2. By Lemma 3.2, we have $\mathbb{P}\left\{\mathcal{S}_{b_{L}, \mathbf{z}}\right\} \leq b_{L}^{-1}|I| q_{L}$, so for the event $\mathcal{S}_{b_{L}}:=\mathcal{S}_{b_{L}, \mathbf{x}} \cup \mathcal{S}_{b_{L}, \mathbf{y}}$ one has $\mathbb{P}\left\{\mathcal{S}_{b_{L}}\right\} \leq 2 b_{L}^{-1}|I|$. For any $\omega \notin \mathcal{S}_{b_{L}}$, each of the two sets $\mathscr{E}_{a_{L}, \mathbf{x}}(\omega), \mathscr{E} a_{L}, \mathbf{y}(\omega)$ has Lebesgue measure bounded by $b_{L}$, thus

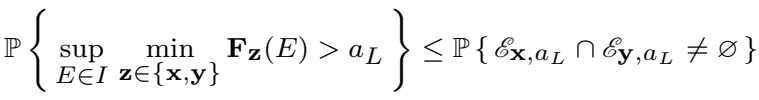

$$
\begin{aligned}
& \leq \mathbb{P}\{\mathcal{S}\}+\mathbb{P}\left\{\left\{\mathscr{E}_{\mathbf{x}, a_{L}} \cap \mathscr{E}_{\mathbf{y}, a_{L}} \neq \varnothing\right\} \backslash \mathcal{S}_{b_{L}}\right\} \\
& \leq 2 b_{L}^{-1}|I|+\mathbb{P}\left\{\left\{\mathscr{E}_{\mathbf{x}, a_{L}} \cap \mathscr{E}_{\mathbf{y}, a_{L}} \neq \varnothing\right\} \backslash \mathcal{S}_{b_{L}}\right\} .
\end{aligned}
$$

By construction of the set $\mathscr{E}_{\mathbf{z}, a_{L}}, \mathbf{z} \in\{\mathbf{x}, \mathbf{y}\}$, for any $\omega \notin \mathcal{S}_{b_{L}}$ it is contained in the $2 c_{L}$-neighborhood of the spectrum $\Sigma_{\mathbf{z}}(\omega)$ of $\mathbf{H}_{\mathrm{B}_{L}(\mathbf{z})}(\omega)$, hence

$$
\begin{aligned}
& \mathbb{P}\left\{\left\{\mathscr{E}_{\mathbf{x}, a_{L}} \cap \mathscr{E}_{\mathbf{y}, a_{L}} \neq \varnothing\right\} \backslash \mathcal{S}_{b_{L}}\right\} \\
& \leq \mathbb{P}\left\{\operatorname{dist}\left(\Sigma_{\mathbf{x}}(\omega), \Sigma_{\mathbf{y}}(\omega)\right) \leq 4 c_{L}\right\} \leq f\left(4 c_{L}\right),
\end{aligned}
$$

by virtue of the eigenvalue comparison bound (3.7). This completes the proof.

It is readily seen that the condition (3.3) is fulfilled with

$$
a_{L}=\mathrm{e}^{-\frac{1}{3} m L}, b_{L}=\mathrm{e}^{-\frac{2}{3} m L}, c_{L}=\mathrm{e}^{-\frac{1}{8} m L}, q_{L}=\mathrm{e}^{-m L},
$$

The proof of the above theorem relies on the EV comparison bound (3.7). For our purposes, it would suffice to quote Ref. [15] where a suitable bound was proved.

Note also that in the context of multi-particle Anderson models in a continuous configuration space $\left(\mathbb{R}^{d}\right)$, Klein and Nguyen [27] recently improved and extended the multiparticle two-volume bound to a large class of alloy-type Anderson Hamiltonians; in particular, this class is larger than the one considered in Refs. [12, 21].

Proposition 3.2 (Cf. [26]*Corollary 2.4). Assume that the marginal probability distribution of the random potential $V$ admits a bounded density $p_{V}$. Then for any pair of $N$-particle configurations $\mathbf{x}, \mathbf{y}$ with $\mathrm{d}_{H}(\mathbf{x}, \mathbf{y})>2 L$, one has

$$
\begin{aligned}
& \mathbb{P}\left\{\operatorname{dist}\left(\Sigma\left(\mathbf{H}_{\mathbf{B}_{L}(\mathbf{x})}\right), \Sigma\left(\mathbf{H}_{\mathbf{B}_{L}(\mathbf{y})}\right)\right) \leq \epsilon\right\} \\
& \leq C(N) L^{2 N d}\left\|p_{V}\right\|_{\infty} \epsilon .
\end{aligned}
$$

The next statement is given in the form presented in [9] and [18], but the credit goes essentially to Germinet and Klein [22] who proved a stronger and more general result. It refers to an interval $I \subset \mathbb{R}$, to be consistent with the previous discussion. However, unlike Theorem 3.1 where integration over $I$ is performed, here the boundedness of $I$ is not important; for example, one could take $I=\mathbb{R}$, provided the validity of the hypothesis (3.10) is established in $I=\mathbb{R}$.

Theorem 3.3 (Cf. [9]*Lemma 9). Given a positive integer $L$, assume that the following bound holds true for a pair of disjoint balls $\mathbf{B}_{L}(\mathbf{x}), \mathbf{B}_{L}(\mathbf{y})$ and some $a_{L}, h_{L}>0$ :

$$
\mathbb{P}\left\{\sup _{E \in I} \min \left[\mathbf{F}_{\mathbf{x}}(E), \mathbf{F}_{\mathbf{y}}(E)\right]>a_{L}\right\} \leq h_{L} .
$$

Then for any finite connected graph $\mathcal{G} \supset \mathbf{B}_{L}(\mathbf{x}) \cup \mathbf{B}_{L}(\mathbf{y})$ one has

$$
\mathbb{E}\left[\sup _{\phi \in \mathscr{B}_{1}(\mathbb{R})}\left|\left\langle\mathbf{1}_{\mathbf{x}}\left|\phi\left(\mathbf{H}_{\boldsymbol{\Lambda}}\right)\right| \mathbf{1}_{\mathbf{y}}\right\rangle\right|\right] \leq 2 a_{L}+h_{L} .
$$

Proof. Fix a finite graph $\mathcal{G} \supset \mathbf{B}_{L}(\mathbf{x}) \cup \mathbf{B}_{L}(\mathbf{y})$. The random operator $\mathbf{H}_{\mathcal{G}}(\cdot)$ has a finite orthonormal basis $\left\{\boldsymbol{\Psi}_{i}(\cdot)\right\}$ with associated EVs $\left\{E_{i}(\cdot)\right\}$. Let

$$
\mathcal{S}_{L}=\left\{\omega: \sup _{E \in I} \min \left[\mathbf{F}_{\mathbf{x}}(E), \mathbf{F}_{\mathbf{y}}(E)\right]>a_{L}\right\} .
$$


By hypothesis, $\mathbb{P}\left\{\mathcal{S}_{L}\right\} \leq h_{L}$. Fix some $\omega \in \mathcal{S}_{L}^{\mathrm{c}}=\Omega \backslash \mathcal{S}_{L}$ and suppose that for each eigenvalue $E_{i}=E_{i}(\omega)$ there is $\mathbf{z}_{i} \in\{\mathbf{x}, \mathbf{y}\}$ such that $\mathbf{B}_{L}\left(\mathbf{z}_{i}\right.$ is $E_{i}$-NS; let $\left\{\mathbf{v}_{i}\right\}=\{\mathbf{x}, \mathbf{y}\} \backslash\left\{\mathbf{z}_{i}\right\}$. Denote $\mathscr{Y}_{\mathbf{x}, \mathbf{y}}:=\left|\left\langle\mathbf{1}_{\mathbf{x}}\left|\phi\left(\mathbf{H}_{\boldsymbol{\Lambda}}\right)\right| \mathbf{1}_{\mathbf{y}}\right\rangle\right|$, then by the GRI for the ball $\mathbf{B}_{L}\left(\mathbf{z}_{i}\right)$ we have, with $\omega$ fixed and omitted from notation for brevity,

$$
\begin{aligned}
\mathscr{Y} \mathbf{x}, \mathbf{y} & \leq\|\phi\|_{\infty} \sum_{E_{i} \in I} \mid \Psi_{i}(\mathbf{x}) \mathbf{\Psi}_{i}\left(\mathbf{y}\left|\leq \sum_{E_{i} \in I}\right| \boldsymbol{\Psi}_{i}\left(\mathbf{v}_{i}\right) \boldsymbol{\Psi}_{i}\left(\mathbf{z}_{i}\right) \mid\right. \\
& \leq \sum_{E_{i} \in I}\left|\mathbf{\Psi}_{i}\left(\mathbf{v}_{i}\right)\right| h_{L}\left(C L^{N d}\right)^{-1} \sum_{\left(\mathbf{u}, \mathbf{u}^{\prime}\right) \in \partial^{-}}\left|\mathbf{\Psi}_{i}(\mathbf{u})\right| \\
& \leq \frac{h_{L}}{C L^{N d}} \sum_{E_{i} \in I}\left|\mathbf{\Psi}_{i}(\mathbf{u})\right| \sum_{\left(\mathbf{u}, \mathbf{u}^{\prime}\right) \in \partial^{-}}\left(\left|\mathbf{\Psi}_{i}(\mathbf{x})\right|+\left|\mathbf{\Psi}_{i}(\mathbf{y})\right|\right) \\
& \leq \frac{h_{L}|\mathbf{B}|}{C L^{N d}} \max _{\mathbf{u} \in \mathcal{G}} \frac{1}{2} \sum_{E_{i} \in I}\left(\left|2 \mathbf{\Psi}_{i}(\mathbf{u})\right|^{2}+\left|\mathbf{\Psi}_{i}(\mathbf{u})\right|+\left|\mathbf{\Psi}_{i}(\mathbf{u})\right|\right) \\
& \leq \frac{h_{L}}{2} \max _{\mathbf{u} \in \mathcal{G}}\left(2\left\|\mathbf{1}_{\mathbf{u}}\right\|^{2}+\left\|\mathbf{1}_{\mathbf{x}}\right\|^{2}+\left\|\mathbf{1}_{\mathbf{y}}\right\|^{2}\right) \leq 2 a_{L},
\end{aligned}
$$

where the last line follows from Bessel's inequality. Hence

$$
\mathbb{E}\left[\mathscr{Y}_{\mathbf{x}, \mathbf{y}}\right] \leq \mathbb{E}\left[\mathbf{1}_{\mathcal{S}_{L}^{\mathrm{c}}} \mathscr{Y} \mathbf{x}, \mathbf{y}\right]+\mathbb{E}\left[\mathbf{1}_{\mathcal{S}_{L}} \mathscr{Y}_{\mathbf{x}, \mathbf{y}}\right] \leq 2 a_{L}+h_{L}
$$

Collecting Theorems 3.1 and 3.3 and Proposition 3.2, we come to the following conclusion.

Theorem 3.4. Suppose that the following condition is fulfilled: for some $R_{0} \in \mathbb{N}$ and all $\mathbb{N} \ni R \geq R_{0}$, for any configurations $\mathbf{x}, \mathbf{y} \in \mathcal{Z}^{N}$ with $\mathrm{d}_{H}(\mathbf{x}, \mathbf{y})>2 R$

$$
\max _{\mathbf{z} \in\{\mathbf{x}, \mathbf{y}\}} \sup _{E \in I} \mathbb{P}\left\{\mathbf{F}_{z}(E)>\mathrm{e}^{-\frac{1}{3} m R}\right\} \leq \mathrm{e}^{-m R} .
$$

Then for any pair of configurations $\mathbf{x}, \mathbf{y} \in \mathcal{Z}^{N}$ with $\mathrm{d}_{H}(\mathbf{x}, \mathbf{y})>2 R \geq 2 R_{0}$ and finite connected subgraph $\mathcal{G} \supset$ $\mathbf{B}_{R}(\mathbf{x}) \cup \mathbf{B}_{R}(\mathbf{y})$ one has

$$
\begin{aligned}
& \mathbb{E}\left[\sup _{\phi \in \mathscr{B}_{1}(\mathbb{R})}\left|\left\langle\mathbf{1}_{\mathbf{x}}\left|\phi\left(\mathbf{H}_{\mathcal{G}}\right)\right| \mathbf{1}_{\mathbf{y}}\right\rangle\right|\right] \\
& \leq\left(C_{1}|I|+C_{2}\right) \mathrm{e}^{-\frac{1}{3} m R}+C_{3} \mathrm{e}^{-\frac{\delta}{8} m R} .
\end{aligned}
$$

Furthermore, on account of the geometrical inequality

$$
\mathrm{d}_{H}(\mathbf{x}, \mathbf{y}) \geq \mathrm{d}_{S}(\mathbf{x}, \mathbf{y})-\operatorname{diam} \Pi \mathbf{x}=\mathrm{d}_{S}(\mathbf{x}, \mathbf{y})-C(\mathbf{x}),
$$

one has, therefore,

$$
\mathbb{E}\left[\sup _{\phi \in \mathscr{B}_{1}(\mathbb{R})}\left|\left\langle\mathbf{1}_{\mathbf{x}}\left|\phi\left(\mathbf{H}_{\mathcal{G}}\right)\right| \mathbf{1}_{\mathbf{y}}\right\rangle\right|\right] \leq \operatorname{Const}(\mathbf{x}) \mathrm{e}^{-\mu \mathrm{d}_{S}(\mathbf{x}, \mathbf{y})} .
$$

The dependence of the RHS factor on $\mathbf{x}$, through diam $\Pi \mathbf{x}$, renders the dynamical localization bound non-uniform in the $N$-particle configuration space, for $N \geq 3$, while for $N=2$ one can actually replace Const(x) by an absolute constant, owing to the equivalence between $\mathrm{d}_{\mathrm{S}}$ and $\mathrm{d}_{\mathcal{H}}$ in this particular case.

\subsection{From EFCs to GFs}

In this subsection we provide a detailed proof of a fairly general relation between the resolvents and the EF correlators, which had been used in numerous works on the FMM. The single- or multi-particle nature of the Hamiltonian at hand is irrelevant, as long as the fractional moments of the EFC can be effectively assessed in the intended application(s) of the general relation. Recall that it suffices for our purposes to establish strong dynamical localization in arbitrarily large but finite domains in the configuration space (with the remaining work to be done with the help of the Fatou lemma, as in $[2,3,4]$ ), so we can indeed restrict our analysis to the finite-dimensional operators.

Lemma 3.3. Let $\mathbf{H}=\mathbf{H}_{\mathcal{G}}$ be an $N$-particle Hamiltonian in a finite graph $\mathcal{G}$, and $\mathbf{G}(\mathbf{x}, \mathbf{y} ; E)$ the kernel of its resolvent $\mathbf{G}(E)=\left(\mathbf{H}_{\Lambda}-E\right)^{-1}$ and $\mathbf{Q}(\mathbf{x}, \mathbf{y})$ the EF correlators (we choose the eigenfunctions real)

$$
\mathbf{Q}(\mathbf{x}, \mathbf{y})=\sum_{i} \boldsymbol{\psi}_{i}(\mathbf{x}) \boldsymbol{\psi}_{i}(\mathbf{y})
$$

Then for any bounded interval $I \subset \mathbb{R}$ and any $s \in(0,1)$

$$
\int_{I}|\mathbf{G}(\mathbf{x}, \mathbf{y} ; E)|^{s} d E \leq \frac{2|I|^{1-s}}{1-s}(\mathbf{Q}(\mathbf{x}, \mathbf{y}))^{s}
$$

Proof. In this deterministic statement, the EFs are fixed, and the only relevant variable is $E$. Fix the points $x$ and $y$. The GF is a rational function, and we divide it into the sum of two terms, according to the signs of the numerators:

$$
\begin{aligned}
& \mathbf{G}(\mathbf{x}, \mathbf{y} ; E)=\sum_{E_{i}: c_{i} \geq 0} \frac{c_{i}}{E_{i}-E}+\sum_{E_{i}: c_{i}<0} \frac{c_{i}}{E_{i}-E} \\
& \left.\left.=: \mathbf{G}^{+} \mathbf{x}, \mathbf{y} ; E\right)+\mathbf{G}^{-} \mathbf{x}, \mathbf{y} ; E\right) .
\end{aligned}
$$

We have

$$
\int_{I}|\mathbf{G}(E)|^{s} d E \leq \int_{I}\left|\mathbf{G}^{+}(E)\right|^{s} d E+\int_{I}\left|\mathbf{G}^{-}(E)\right|^{s} d E
$$

Both integrals are assessed in the same way, so we focus on the first one.

It is convenient at this point to introduce probabilistic language, as we are going to apply a standard technique for the probability distribution functions (PDF), and consider the probability space $\left(I, \mathfrak{B}_{I}\right.$, mes$\left._{I}\right)$, where $\mathfrak{B}_{I}$ is the Borel sigmaalgebra and $\operatorname{mes}_{I}:=|I|^{-1}$ mes the normalized (i.e., probability) Lebesgue measure in $I \subset \mathbb{R}$. Further, consider the measurable function $\mathbf{G}^{ \pm}: E \mapsto \mathbf{G}^{ \pm}(E)$ (i.e., a "random variable" on $\left(I, \operatorname{mes}_{I}\right)$ ), and let $F_{ \pm}(t)$ be the PDF of its absolute value:

$$
\left.F_{ \pm}(t)=\operatorname{mes}_{I}\left\{E: \mid \mathbf{G}^{ \pm} \mathbf{x}, \mathbf{y} ; E\right) \mid \leq t\right\} .
$$

Then

$$
\begin{aligned}
& \int_{I}\left|\mathbf{G}^{ \pm}(\mathbf{x}, \mathbf{y} ; E)\right|^{s} d E=|I| \widehat{\mathbb{E}}^{I}\left[\left|\mathbf{G}^{ \pm}(\mathbf{x}, \mathbf{y} ; E)\right|^{s}\right] \\
& =|I| \int_{0}^{\infty} t^{s} d F_{ \pm}(t) .
\end{aligned}
$$

Using integration by parts for the Stiltjes integral, we obtain for any $s>0$

$$
\int_{0}^{\infty} t^{s} d F_{ \pm}(t)=s \int_{0}^{\infty} t^{s-1}\left(1-F_{ \pm}(t)\right) d t
$$

where both integrals converge or diverge simultaneously. The goal of this transformation is to reduce the estimate to that of the tail distribution function $t \mapsto 1-F_{ \pm}(t)=\operatorname{mes}_{I}\{E$ : $\left.\mathbf{G}^{ \pm}(E)>t\right\}$, with the help of the Boole identity (cf. Proposition C.1), applicable to any rational function with simple, real poles and positive expansion coefficients, $f: t \mapsto \sum_{i=1}^{n} \frac{c_{i}}{t_{i}-t}$, and stating that

$$
\operatorname{mes}\{\lambda:|f(\lambda)|>t\}=\frac{2 \sum_{i=1}^{n} c_{i}}{t},
$$


hence

$$
\operatorname{mes}_{I}\{\lambda:|f(\lambda)|>t\} \leq \frac{2 \sum_{i=1}^{n} c_{i}}{|I| t},
$$

Recall that, in fact, $c_{i}=\psi_{i}(\mathbf{x}) \psi_{i}(\mathbf{y})$ (we choose the EFs real), so that

$$
\sum_{i: c_{i} \geq 0} c_{i} \leq \mathbf{Q}_{+}(\mathbf{x}, \mathbf{y}), \sum_{i: c_{i}<0}\left(-c_{i}\right) \leq \mathbf{Q}_{-}(\mathbf{x}, \mathbf{y}),
$$

where $\mathbf{Q}_{ \pm}(\mathbf{x}, \mathbf{y})$ are components of the EF correlator:

$$
\mathbf{Q}_{+}(\mathbf{x}, \mathbf{y})+\mathbf{Q}_{-}(\mathbf{x}, \mathbf{y})=\sum_{i: E_{i} \in I}\left|c_{i}\right| \leq \mathbf{Q}(\mathbf{x}, \mathbf{y})
$$

Thus, denoting for brevity $\mathbf{Q}_{ \pm} \equiv \mathbf{Q}_{ \pm}(\mathbf{x}, \mathbf{y})$, we have

$$
\begin{aligned}
& 1-F_{ \pm}(t) \leq \min \left(1,2 \mathbf{Q}_{ \pm}(|I| t)^{-1}\right) \\
& =\mathbf{1}_{\left[0,2 \mathbf{Q}_{ \pm} /|I|\right]}(t)+\frac{2 \mathbf{Q}_{ \pm}}{|I| t} \mathbf{1}_{\left[2 \mathbf{Q}_{ \pm} /|I|,+\infty\right)}(t)
\end{aligned}
$$

and

$$
\begin{aligned}
\int_{0}^{\infty} t^{s} d F_{ \pm}(t) & \leq s \int_{0}^{\infty} t^{s-1}\left(1-F_{ \pm}(t)\right) d t \\
& \leq s \int_{0}^{2 \mathbf{Q} /|I|} t^{s-1} d t+2 s \mathbf{Q}_{ \pm} \int_{2 \mathbf{Q} /|I|}^{\infty} t^{s-2} d t \\
& =\left(\frac{2 \mathbf{Q}_{ \pm}}{|I|}\right)^{s}+2 s \mathbf{Q}_{ \pm} \frac{\left(2 \mathbf{Q}_{ \pm}\right)^{s-1}}{|I|^{s}(1-s)} \\
& =\frac{\left(2 \mathbf{Q}_{ \pm}\right)^{s}|I|^{-s}}{1-s} .
\end{aligned}
$$

Therefore, using $\frac{\alpha^{s}+\beta^{s}}{2} \leq\left(\frac{\alpha+\beta}{2}\right)^{s}, s<1$, we obtain

$$
\begin{aligned}
\int_{I}|\mathbf{G}(\mathbf{x}, \mathbf{y} ; E)|^{s} d E & \left.\left.\leq|I| \cdot \frac{2^{s}|I|^{-s}}{1-s}\left(\mathbf{Q}_{+}(\mathbf{x}, \mathbf{y})\right)^{s}+\mathbf{Q}_{-}(\mathbf{x}, \mathbf{y})\right)^{s}\right) \\
& \leq \frac{2(\mathbf{Q}(\mathbf{x}, \mathbf{y}))^{s}|I|^{1-s}}{1-s}
\end{aligned}
$$

\section{Decay of the fractional moments of the GFs}

Following [5], we will use the sequence of length scales $\left\{L_{k}, k \geq 0\right\}$ defined by

$$
L_{k+1}:=2\left(L_{k}+1\right), \quad k=0,1, \ldots,
$$

or, explicitly,

$$
L_{k}=2^{k}\left(L_{0}+2\right)-2 .
$$

We consider two $N$-particle configurations $\mathbf{x}, \mathbf{y}$ with $R:=$ $\mathrm{d}_{\mathcal{H}}(\mathbf{x}, \mathbf{y}) \in\left(L_{k}, L_{k+1}\right]$ for some $k \in \mathbb{N}$, and choose some $a \in \Pi \mathbf{x}, y \in \Pi \mathbf{y}$ such that $R=\mathrm{d}(a, y)$. Such points $a, y$ exist by definition of the Hausdorff distance.

On Fig. 1, where the case $\mathcal{Z}=\mathbb{Z}^{1}$ is illustrated, we set $a=0$, by translation invariance of the random potential in the 1-particle configuration space.

Introduce the following notation:

$$
\mathcal{X}_{L}^{\Lambda}(u)=\mathcal{X}_{L}^{\Lambda, N}(u)=\left\{\mathbf{x} \in \Lambda^{N}: \Pi \mathbf{x} \ni u, \operatorname{diam} \Pi \mathbf{x} \leq L\right\} .
$$

Equivalently, setting $\mathbf{u}=(u, u, \ldots, u), \mathcal{X}_{L}^{\Lambda}(u)=\Lambda_{L}(\mathbf{u}) \cap \Lambda^{N}$ is the set of all $N$-particle configurations in the "physical" domain $\Lambda$ at distance $\leq L$ from the position $u$.

For notational brevity, the Green functions $\mathbf{G}(\mathbf{x}, \mathbf{y})$ with no subscript (and the energy $E$ usually omitted from notation, as we perform a fixed-energy analysis in the scaling procedure) refer to the Hamiltonian in the above mentioned large - and fixed - domain $\Lambda$.

In order to successfully carry out the scale induction, one needs of course some technical assumptions relative to the fractional moments and eigenfunction correlators at a (properly chosen) initial scale $L_{0}$. Such initial scale estimates will appear in the course of the induction step; their validity at the scale $L_{0}$ is discussed in Section 5 below.

As to the magnitude of $L_{0}$ suitable for the induction, it can be arbitrary for the proof of localization at large disorder: the larger $L_{0}$, the larger must be the amplitude $|g|$ of the random potential guaranteeing the onset of the $\mathrm{N}$-particle localization. The strong disorder manifests itself in a very clear way - through a small constant $M_{s} \leq C|g|^{-s}$ figuring in a number of formulae.

However, in the case of an $N$-particle system in one dimension, with possibly small disorder amplitude $|g|$, one has to choose $L_{0}$ large enough, to ensure that the decay of GFs/EFs/EFCs at distance $L_{0}$ is perceptible. See a brief discussion of the Lyapunov exponents in one dimension in Section 5.2.

\section{Assumption for the step $N-1 \rightsquigarrow N, N \geq 2$.}

For all $n \in[1, N-1]$, the EF correlators $\mathbf{Q}^{(n)}(\mathbf{x}, \mathbf{y})$ of $n$-particle systems in the domain $\Lambda$ fulfill the following condition: for some $A, m_{N-1}>0$ and all $\mathbf{x}, \mathbf{y} \in \mathcal{X}_{L}^{\Lambda, n}$

$$
\mathbb{E}\left[\mathbf{Q}^{(n)}(\mathbf{x}, \mathbf{y})\right] \leq A \mathrm{e}^{-m_{N-1} \mathrm{~d}_{\mathcal{H}}(\mathbf{x}, \mathbf{y})} .
$$

Observe that the expectation in (4.4) is relative only to the disorder, since the EFCs do not depend upon energy.

The rest of this section is the central part of the paper. Its role and relations with other sections can be described as follows.

1. Below we focus on the derivation of exponential decay bounds on the fractional moments of the $N$-particle system in $\Lambda$, with some $m_{N} \in\left(0, m_{N-1}\right)$ to be chosen appropriately.

2. The validity of the assumption (4.4) for $N=2$, i.e., relative to the 1-particle systems, is discussed in Section 5. It follows from the well-known results of the singleparticle fractional moment analysis.

3. Once the exponential decay bounds on the fractional moments of the $N$-particle GFs are established, the corresponding exponential bounds on the $N$-particle EFCs (required for the next step of the induction on the number of particles, $N \rightsquigarrow N+1$ ) will follow from the general arguments given in Sect. 3.2. An important (and unfortunate) feature of the existing methods of the multi-particle Anderson localization theory, including the MPFMM, is a deterioration of the decay rate $m_{n}$ at each induction step $n \rightsquigarrow n+1$ :

$$
m_{1}>m_{2} \cdots>m_{N-1}>m_{N}
$$

In fact, both the MPMSA and the MPFMM result in $m_{N} \sim$ $O\left(m_{1} /(N-1) !\right)$.

For notational brevity, below we often drop the subscripts from the notations for the decay rates like $m_{n}$. We work here with the $N$-particle systems and their subsystems of $n \leq N-1$ particles in $\Lambda$, so the minimal decay rate is given by $m_{N-1}>$ 0 which we denote by $m$. 
Theorem 4.1. Fix $N \geq 2$ and assume that for all $n=1, \ldots$, $N-1$, the exponential decay of theEFCs is established, in the Hausdorff distance(s) for n-particle Hamiltonians $\mathbf{H}^{(n)}(\omega)$ with decay exponents $m_{1}>m_{2}>\cdots>m_{n-1}$. Then for some $c>0$, the $N$-particle Hamiltonian $\mathbf{H}^{(N)}(\omega)$ also feature exponential decay of the EFCs in the Hausdorff distance, with decay exponent $m_{N} \geq \mathrm{cm}_{N-1} /(N-1)$.

Proof. The argument given below relies on Theorem 4.2. The case where max $[\operatorname{diam} \mathbf{x}, \operatorname{diam} \mathbf{y}]>L_{k} / 2$ can be analyzed separately, and we do so in Section 6. By Lemma 6.1, the assumption diam $\mathbf{x}>L_{k} / 2$ along with (4.1) and the inductive hypothesis (4.4) imply

$$
\mathbb{E}_{I}\left[|\mathbf{G}(\mathbf{x}, \mathbf{y})|^{s}\right] \leq A \mathrm{e}^{-c_{N} m L_{k}}=A \mathrm{e}^{-\frac{c m_{N}-1}{N-1} L_{k}}, c>0 .
$$

Hence we can focus on the pairs of configurations of diameter $\leq L_{k} / 2$.

Since $\Pi \mathbf{x} \ni a$ and diam $\mathbf{x} \leq L_{k} / 2$, we have $\mathbf{x} \in \mathbf{B}_{L_{k} / 2}(\mathbf{a})$, where we denote $\mathbf{a}=(a, a, \ldots, a)$. By construction,

$$
\min _{i} \mathrm{~d}\left(x_{i}, y\right)=\mathrm{d}(\Pi \mathbf{x}, y)=\mathrm{d}_{\mathcal{H}}(\mathbf{x}, \mathbf{y})=R>L_{k},
$$

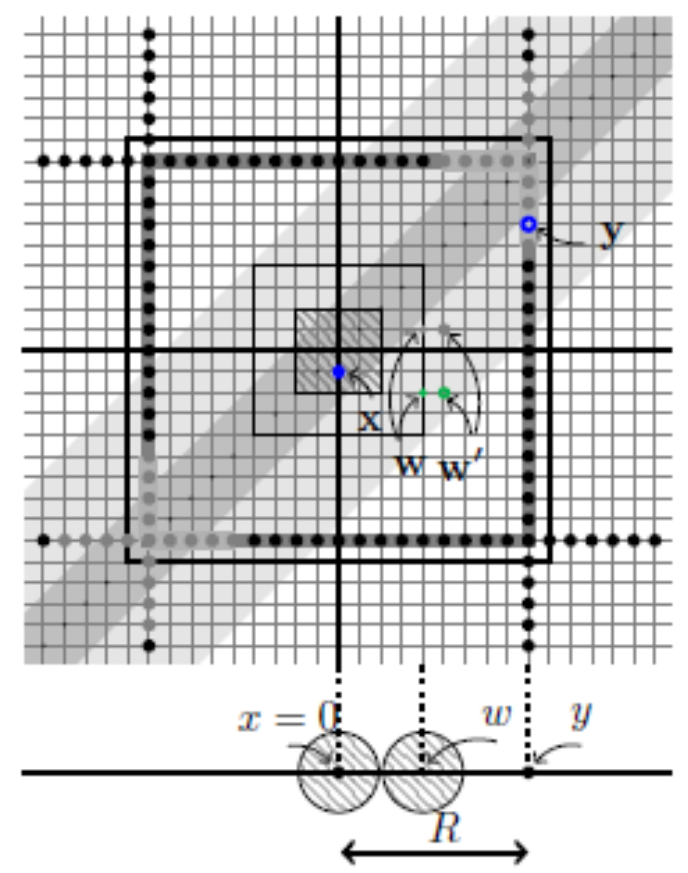

Figure 1. An example for the proof of Theorem 4.1. Here $d=1, N=2$.

thus $y \notin \Pi B_{L_{k} / 2}(\mathbf{a})$. By the FGRI applied to the cube $\mathbf{B}_{L_{k} / 2}(\mathbf{a})$,

$$
\begin{aligned}
& \widehat{\mathbb{E}}^{I}\left[|\mathbf{G}(\mathbf{x}, \mathbf{y})|^{s}\right] \leq\left|\partial \mathbf{B}_{L_{k} / 2}(\mathbf{a})\right| . \\
& \max _{\left(\mathbf{w}, \mathbf{w}^{\prime}\right) \in \partial \mathbf{B}_{L_{k} / 2}(\mathbf{a})} \widehat{\mathbb{E}}^{I}\left[\left|\mathbf{G}_{\mathbf{B}_{L_{k} / 2}(\mathbf{a})}(\mathbf{x}, \mathbf{w})\right|^{s}\left|\mathbf{G}\left(\mathbf{w}^{\prime}, \mathbf{y}\right)\right|^{s}\right] .
\end{aligned}
$$

Here $\Pi \mathbf{w}^{\prime}=\left\{w^{\prime}, w_{2}^{\prime}, \ldots, w_{N-1}^{\prime}\right\}$ with $\mathrm{d}\left(0, w^{\prime}\right)=\frac{1}{2} L_{k}+1$. Set $u_{1}=w^{\prime}, u_{2}=y$. Then $u_{1}, u_{2} \notin \Lambda_{L_{k} / 2}(0)$, so $\mathbf{G}_{\mathbf{B}_{L_{k} / 2}(\mathbf{a})}(\mathbf{x}, \mathbf{w})$ is $\mathfrak{F}_{\neq u_{1}, u_{2}}$-measurable, with $\mathfrak{F}_{\neq u_{1}, u_{2}}$ generated by $\left\{V(z ; \cdot), z \in \Omega \backslash\left\{u_{1}, u_{2}\right\}\right\}$, so for any fixed pair $\mathbf{w}, \mathbf{w}^{\prime}$ as above,

$$
\begin{aligned}
& \widehat{\mathbb{E}}^{I}\left[\left|\mathbf{G}_{\mathbf{B}_{L_{k} / 2}(\mathbf{a})}(\mathbf{x}, \mathbf{w})\right|^{s}\left|\mathbf{G}\left(\mathbf{w}^{\prime}, \mathbf{y}\right)\right|^{s}\right] \\
& \leq \widehat{\mathbb{E}}^{I}\left[\left|\mathbf{G}_{\mathbf{B}_{L_{k} / 2}(\mathbf{x})}(\mathbf{x}, \mathbf{w})\right|^{s} \widehat{\mathbb{E}}^{I}\left[\left|\mathbf{G}\left(\mathbf{w}^{\prime}, \mathbf{y}\right)\right|^{s} \mid \mathfrak{F}_{\Omega \backslash\left\{u_{1}, u_{2}\right\}}\right]\right] .
\end{aligned}
$$

Applying Lemma 3.1 (cf. Eqn. (3.1)), we obtain a uniform upper bound

$$
\widehat{\mathbb{E}}^{I}\left[\left|\mathbf{G}\left(\mathbf{w}^{\prime}, \mathbf{y}\right)\right|^{s} \mid \mathfrak{F}_{\Omega \backslash\left\{u_{1}, u_{2}\right\}}\right] \leq C_{s}|g|^{-s},
$$

thus

$$
\begin{aligned}
& \widehat{\mathbb{E}}^{I}\left[|\mathbf{G}(\mathbf{x}, \mathbf{y})|^{s}\right] \\
& \leq C_{s}|g|^{-s}\left|\partial \mathbf{B}_{L_{k} / 2}(\mathbf{a})\right| \widehat{\mathbb{E}}^{I}\left[\left|\mathbf{G}_{\mathbf{B}_{L_{k} / 2}(\mathbf{x})}(\mathbf{x}, \mathbf{w})\right|^{s}\right] .
\end{aligned}
$$

Aplying the bound (4.13), we conclude that

$$
\widehat{\mathbb{E}}^{I}\left[|\mathbf{G}(\mathbf{x}, \mathbf{y})|^{s}\right] \leq C_{s}|g|^{-s}\left|\partial \mathbf{B}_{L_{k} / 2}(\mathbf{a})\right| \mathrm{e}^{-c m L_{k}} .
$$

Assessing the RHS expectation in (4.9) is the most tedious task, and it is entrusted to the multi-scale induction in Theorem 4.2. Introduce the following notations, to be used below with $L=L_{j+1}$ ) (cf. (4.3):

$$
\Upsilon(L):=\left|\partial \mathrm{B}_{L}(a)\right| \sup _{\Lambda \subseteq \mathrm{B}_{L}(a)} \sum_{\substack{\mathrm{d}(a, u)=L \\ \mathbf{x} \in \mathcal{X}_{L / 2}^{\Lambda}(a) \\ \mathbf{w} \in \mathcal{X}_{L / 2}^{\Lambda}(w)}} \widehat{\mathbb{E}}^{I}\left[\left|\mathbf{G}_{\Omega}(\mathbf{x}, \mathbf{w})\right|^{s}\right] .
$$

We also need a slightly modified quantity, defined for $L=$ $L_{j+1}, j \geq 0$ :

$$
\widetilde{\Upsilon}\left(L_{j+1}\right):=\left|\partial \mathrm{B}_{L_{j+1}}(a)\right| \sup _{\Lambda \subseteq \mathrm{B}_{L_{j+1}}} \sum_{\substack{\mathrm{d}(a, u)=L_{j+1} \\ \mathbf{x} \in \mathcal{X}_{L_{j} / 2}^{\Lambda}(a) \\ \mathbf{w} \in \mathcal{X}_{L_{j} / 2}^{\Lambda}(w)}} \widehat{\mathbb{E}}^{I}\left[\left|\mathbf{G}_{\Lambda}(\mathbf{x}, \mathbf{w})\right|^{s}\right] .
$$

The correlators $\Upsilon\left(L_{j}\right), \Upsilon\left(L_{j+1}\right)$ are required to carry out the scale induction, but $\widetilde{\Upsilon}\left(L_{j+1}\right)$ are simpler to assess.

Theorem 4.2. For all $k \geq 0$,

$$
\Upsilon\left(L_{k}\right) \leq \mathrm{e}^{-c m L_{k}} .
$$

Proof. Unlike Theorem 4.1, we consider now the pairs $\mathbf{x}, \mathbf{y}$ with $\Pi \mathbf{x} \ni a$, $\Pi \mathbf{w} \ni w$ with specific values of the distance $\mathrm{d}(a, w)=L_{j}, j \geq 1$, so the scale induction will be carried out only for such distances. To avoid confusion with the previously used notation and arguments, the scales will be labeled by the index $j$.

We have $\mathbf{x} \in \mathbf{B}_{L_{j} / 2}(\mathbf{a}) \subset \mathbf{B}_{L_{j}}(\mathbf{a}), \mathbf{w} \in \partial^{-} \mathbf{B}_{L_{j}}(\mathbf{a})$. Here $\mathbf{w}$ is $L_{j} / 2$-split and distant from $\mathbf{x}$ : $\operatorname{diam} \mathbf{w}>L_{j} / 2, \mathrm{~d}_{\mathrm{S}}(\mathbf{x}, \mathbf{w}) \leq$ $L_{j} / 2$, thus by Lemma 6.1,

$$
\widehat{\mathbb{E}}^{I}\left[\left|\mathbf{G}_{\mathbf{B}_{L}(\mathbf{a})}(\mathbf{x}, \mathbf{w})\right|^{s}\right] \leq \text { Const } \mathrm{e}^{-c m L_{j}}
$$

From this point on, we consider the configurations $w$ of restricted diameter.

We aim to show first that the quantities $\Upsilon\left(L_{j}\right), j \geq 0$, satisfy the recursion

$$
\Upsilon\left(2\left(L_{j}+1\right)\right) \leq \frac{A^{\prime}}{|g|^{s}} \Upsilon^{2}\left(L_{j}\right)+A L_{j+1}^{2 q} \mathrm{e}^{-2 \nu L_{j}},
$$

and then infer from (4.14) that $\Upsilon\left(L_{j}\right)$ decay exponentially.

Approximation by truncated correlators. Let us show that (cf. (4.11)-(4.12))

$$
0 \leq \Upsilon\left(L_{j+1}\right)-\widetilde{\Upsilon}\left(L_{j+1}\right) \leq 2 A \cdot\left(L_{j+1}\right)^{2} \mathrm{e}^{-c m L_{j}} .
$$

Consider any term $\widehat{\mathbb{E}}^{I}\left[\left|\mathbf{G}_{\Lambda}(\mathbf{x}, \mathbf{w})\right|^{s}\right]$ figuring in the sum for $\Upsilon\left(L_{j+1}\right)$ but absent in $\widetilde{\Upsilon}\left(L_{j+1}\right)$. Its exclusion from $\widetilde{\Upsilon}\left(L_{j+1}\right)$ implies that

$$
\frac{1}{2} L_{j}<\operatorname{diam} \mathbf{w} \leq \frac{1}{2} L_{j+1}
$$


here the RHS inequality is due to the constraint figuring in the definition of $\Upsilon\left(L_{j+1}\right)$. Both bounds on diam $\mathbf{w}$ are important. First, $\Pi \mathbf{x} \ni a$ and $\Pi \mathbf{w} \ni w$ with $\mathrm{d}(a, w)=L_{j+1}$, so it follows from the upper bound diam $\mathbf{x} \leq L_{j+1} / 2$ that

$$
\mathrm{d}_{\mathcal{H}}(\mathbf{x}, \mathbf{w}) \geq \mathrm{d}(a, u)-\frac{1}{2} L_{j+1}=\frac{1}{2} L_{j+1} .
$$

Next, the lower bound diam $\mathbf{w}>L_{j} / 2$ enables us to apply Lemma 6.1 on $R$-distant configurations at least one of which is $R$-split; here we have $R=L_{j} / 2$ with $L_{j}=\frac{1}{2} L_{j+1}-1 \geq$ $\frac{1}{3} L_{j+1}$, thus

$$
\widehat{\mathbb{E}}^{I}\left[\left|\mathbf{G}_{\Lambda}(\mathbf{x}, \mathbf{w})\right|^{s}\right] \leq A \mathrm{e}^{-c m L_{j}}=A \mathrm{e}^{-\frac{c}{3} m L_{j+1}},
$$

so it remains only to assess the number of relevant terms.

There are at most $C L_{j+1}^{2(N-1) d}$ choices for the pair $(\mathbf{x}, \mathbf{w})$, since $\Pi \mathbf{w} \ni u$ with $u$ fixed, $\Pi \mathbf{x} \ni a$, and $\operatorname{diam} \mathbf{x}$, diam $\mathbf{w} \leq$ $\frac{1}{2} L_{j+1}$. Thus the number of terms which constitute the difference $\Upsilon\left(L_{j+1}\right)-\widetilde{\Upsilon}\left(L_{j+1}\right)$ is bounded by $C L_{j+1}^{2(N-1) d}$. This completes the proof of (4.15).

Recursion with truncated correlators. Consider the correlator $\widetilde{\Upsilon}\left(L_{j+1}\right)$. Introduce the configuration $\widehat{\mathbf{w}}=(w, w, \ldots, w)$ (it plays the role similar to that of $\mathbf{a}=(a, a, \ldots, a)$, and denote for brevity $\mathbf{B}^{\prime}=\mathbf{B}_{L_{j}}(\mathbf{a}), \mathbf{B}^{\prime \prime}=\mathbf{B}_{L_{j}}(\widehat{\mathbf{w}}), \Lambda^{\prime}=\mathrm{B}_{L_{j} / 2}(0)$, $\Lambda^{\prime \prime}=\mathrm{B}_{L_{j} / 2}(u)$. By the FGRI, we can write

$$
\begin{aligned}
& \sum_{\substack{\left.\mathbb{E}^{I}, \mathbf{z}^{\prime}\right\rangle \in \partial \mathbf{B}_{L_{j}}(\mathbf{a}) \\
\left\langle\mathbf{v}, \mathbf{v}^{\prime}\right\rangle \in \partial \mathbf{B}_{L_{j}}(\mathbf{w})}}^{\mathbb{E}\left[\left|\mathbf{G}_{\mathbf{B}^{\prime}}(\mathbf{x}, \mathbf{z})\right|^{s}\left|\mathbf{G}_{\mathbf{B}^{\prime \prime}}(\mathbf{v}, \mathbf{w})\right|^{s} .\right.} \\
& \left.\cdot \widehat{\mathbb{E}}^{I}\left[\left|\mathbf{G}\left(\mathbf{z}^{\prime}, \mathbf{v}^{\prime}\right)\right|^{s} \mid \mathfrak{F}_{\Lambda^{\prime} \cup \Lambda^{\prime \prime}}\right]\right]
\end{aligned}
$$

aiming, of course, to obtain a uniform upper bound on the conditional expectation in the RHS. To this end, notice that $\mathrm{d}\left(\mathbf{z}^{\prime}, \mathbf{a}\right)=1+L_{j} / 2$, thus $\Pi \mathbf{z}^{\prime} \ni z^{\prime}$ with $z^{\prime} \notin \Lambda^{\prime}$. Similarly, $\Pi \mathbf{v}^{\prime} \ni v^{\prime}$ with $v^{\prime} \notin \Lambda^{\prime \prime}$.

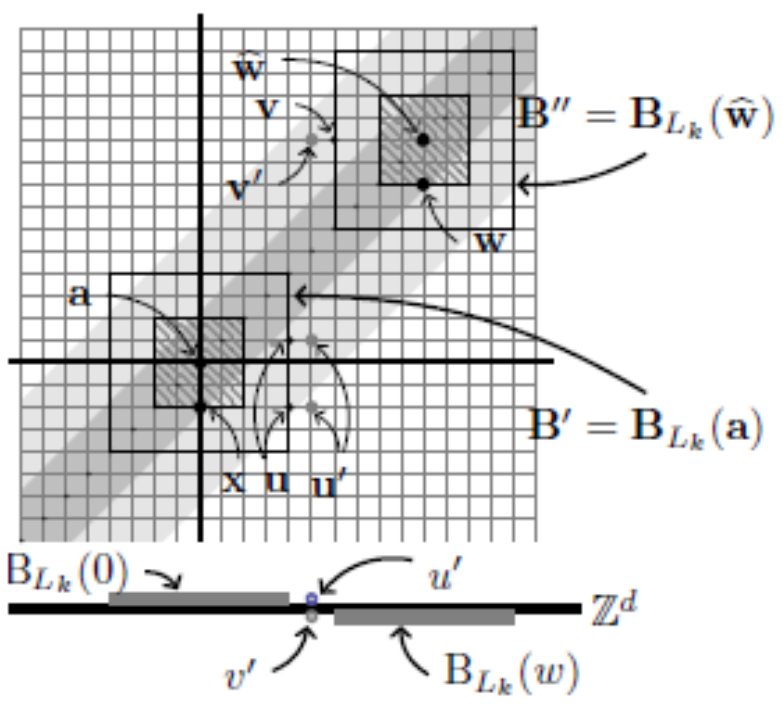

Therefore, the sigma-sub-algebra $\mathfrak{F}_{\neq z^{\prime}, v^{\prime}}$ generated by the random potential in $\mathcal{Z} \backslash\left\{z^{\prime}, v^{\prime}\right\}$ is larger than $\mathfrak{F}_{\Lambda^{\prime} \cup \Lambda^{\prime \prime}}$, and applying Lemma 3.1 to the expectation $\widehat{\mathbb{E}}^{I}\left[\cdot \mid \mathfrak{F}_{\neq z^{\prime}, v^{\prime}}\right]$, we obtain

$$
\begin{aligned}
& \widehat{\mathbb{E}}^{I}\left[\left|\mathbf{G}\left(\mathbf{z}^{\prime}, \mathbf{v}^{\prime}\right)\right|^{s} \mid \mathfrak{F}_{\Lambda^{\prime} \cup \Lambda^{\prime \prime}}\right] \\
& =\mathbb{E}_{I}\left[\mathbb{E}_{I}\left[\left|\mathbf{G}\left(\mathbf{z}^{\prime}, \mathbf{v}^{\prime}\right)\right|^{s} \mid \mathfrak{F}_{\neq z^{\prime}, v^{\prime}}\right] \mid \mathfrak{F}_{\Lambda^{\prime} \cup \Lambda^{\prime \prime}}\right] \leq \frac{C}{g^{s}} .
\end{aligned}
$$

Thus

$$
\begin{aligned}
& \widehat{\mathbb{E}}^{I}\left[|\mathbf{G}(\mathbf{x}, \mathbf{w})|^{s}\right] \\
& \leq \frac{C}{g^{s}} \sum_{\substack{\left\langle\mathbf{z}, \mathbf{z}^{\prime}\right\rangle \in \partial \mathbf{B}_{L_{j}}(\mathbf{a}) \\
\left\langle\mathbf{v}, \mathbf{v}^{\prime}\right\rangle \in \partial \mathbf{B}_{L_{j}}(\mathbf{w})}} \widehat{\mathbb{E}}^{I}\left[\left|\mathbf{G}_{\mathbf{B}^{\prime}}(\mathbf{x}, \mathbf{u})\right|^{s}\right] \widehat{\mathbb{E}}^{I}\left[\left|\mathbf{G}_{\mathbf{B}^{\prime \prime}}(\mathbf{v}, \mathbf{w})\right|^{s}\right] .
\end{aligned}
$$

Now consider $\widehat{\mathbb{E}}^{I}\left[\left|\mathbf{G}_{\mathbf{B}^{\prime}}(\mathbf{x}, \mathbf{u})\right|^{s}\right]$; the remaining expectation in the RHS is assessed similarly. This is done in two steps:

1. For $\mathbf{z}$ with diam $\mathbf{u} \leq L_{j} / 2$ we use the scale induction:

$$
\sum_{\substack{\left\langle\mathbf{z}, \mathbf{z}^{\prime}\right\rangle \in \partial \mathbf{B}_{L_{j}}(\mathbf{a}) \\ \operatorname{diam} \mathbf{u} \leq L_{j} / 2}} \widehat{\mathbb{E}}^{I}\left[\left|\mathbf{G}_{\boldsymbol{\Lambda}^{\prime}}(\mathbf{x}, \mathbf{z})\right|^{s}\right] \leq \Upsilon\left(L_{j}\right) .
$$

2. If $\operatorname{diam} \mathbf{z}>L_{j} / 2$, then we apply Lemma 6.1:

$$
\widehat{\mathbb{E}}^{I}\left[\left|\mathbf{G}_{\boldsymbol{\Lambda}^{\prime}}(\mathbf{x}, \mathbf{z})\right|^{s}\right] \leq A \mathrm{e}^{-\frac{m}{2} L_{j}} .
$$

Collecting all possible vertices $\mathbf{z}$ of the categories (1) and (2), and upper-bounding the number of vertices from each category by $\left|\partial^{-} \boldsymbol{\Lambda}(\mathbf{x})\right|$, we obtain

$$
\sum_{\mathbf{z}} \widehat{\mathbb{E}}^{I}\left[\left|\mathbf{G}_{\boldsymbol{\Lambda}^{\prime}}(\mathbf{x}, \mathbf{z})\right|^{s}\right] \leq C\left(\Upsilon\left(L_{j}\right)+C^{\prime} L_{j}^{q} \mathrm{e}^{-m L_{j}}\right),
$$

with $q=2(N-1) d$. Similarly,

$$
\sum_{\mathbf{v}} \widehat{\mathbb{E}}^{I}\left[\left|\mathbf{G}_{\boldsymbol{\Lambda}^{\prime \prime}}(\mathbf{v}, \mathbf{w})\right|^{s}\right] \leq C|g|^{-s}\left(\Upsilon\left(L_{j}\right)+C^{\prime} L_{j}^{q} \mathrm{e}^{-\frac{m}{2} L_{j}}\right) .
$$

Denote $M_{s}=2 C|g|^{-s}$, then

$$
\widetilde{\Upsilon}\left(L_{j+1}\right) \leq \frac{1}{2} M_{s}\left(\Upsilon\left(L_{j}\right)+C^{\prime} L_{j}^{q} \mathrm{e}^{-\frac{m}{2} L_{j}}\right)^{2} .
$$

where

Applying the approximation formula (4.15), we obtain

$$
\begin{aligned}
\Upsilon\left(L_{j+1}\right) & \leq \frac{1}{2} M_{s}\left(\Upsilon\left(L_{j}\right)+C^{\prime} \mathrm{e}^{-\frac{m}{2} L_{j}}\right)^{2}+A L_{j+1}^{p} \mathrm{e}^{-\frac{m}{2} L_{j}} \\
& \leq \frac{1}{2} M_{s}\left(\Upsilon\left(L_{j}\right)+\mathrm{e}^{-\frac{m}{3} L_{j}}\right)^{2}+\frac{1}{2} \mathrm{e}^{-\frac{m}{3} L_{j}},
\end{aligned}
$$

provided $L_{0}$ is large enough (depending on $m>0$ ).

Let $\widehat{\Upsilon}_{j}=\Upsilon\left(L_{j}\right)+\mathrm{e}^{-\frac{m}{3} L_{j}}$, then (4.16) can be re-written as follows:

$$
\widehat{\Upsilon}_{j+1} \leq \frac{1}{2} M_{s} \widehat{\Upsilon}_{j}^{2}+\frac{1}{2} \mathrm{e}^{-\frac{m}{4} L_{j}} .
$$

In order to complete the induction step, we have to assume that

$$
M_{s}^{1 / 2} \widehat{\Upsilon}_{0}<1
$$

thus

$$
\exists \nu>0: \quad \beta_{0}:=\mathrm{e}^{2 \nu} M_{s}^{1 / 2} \widehat{\Upsilon}_{0} \leq \mathrm{e}^{-\nu L_{0}} .
$$

The validity of the assumption (4.18) (hence, that of (4.19)) is established in Section 5: (i) for strongly disordered systems, and (ii) for sufficiently weak perturbations of localized noninteracting systems in one dimension.

Denote $\beta_{k}=\mathrm{e}^{2 \nu} M_{s}^{1 / 2} \widehat{\Upsilon}_{k}, k \geq 0$, then we have the recursion

$$
\begin{aligned}
\beta_{k+1} & \leq \frac{1}{2} \mathrm{e}^{-2 \nu} \beta_{k}^{2}+\frac{1}{2} \mathrm{e}^{2 \nu} M_{s}^{1 / 2} \mathrm{e}^{-\frac{m}{4} L_{k}} \\
& \leq \frac{1}{2} \mathrm{e}^{-2 \nu} \beta_{k}^{2}+\frac{1}{2} \mathrm{e}^{-\frac{m}{5} L_{k}},
\end{aligned}
$$

provided that $L_{0}$ is large enough. A simple calculation (cf. Lemma A.1) shows that (4.20) implies

$$
\forall k \geq 0 \quad \Upsilon\left(L_{k}\right) \leq \text { Const } \mathrm{e}^{-\widetilde{\mu} L_{k}},
$$


with some $\widetilde{\mu} \geq \mathrm{cm}>0$ (specified in Lemma A.1). This completes Step 4 and the proof of exponential decay of the fractional moments of the $N$-particle Green functions. As was explained before, the output from the induction step $N-1 \rightsquigarrow N$ for the GFs implies exponential decay of the $N$-particle EFCs (cf. Sect. 3.2). This makes possible the next induction step $N \rightsquigarrow N+1$, but only if the initial decay rate $m_{1}$ (cf. (4.5)) is large enough, or, more to the point, if the obtained decay rate $m_{N}$ for the $N$-particle systems is not too small - this is why the induction in $N$ cannot be continued indefinitely.

\section{Initial scale estimates for the $N$ - parictle system}

\subsection{Strongly disordered systems in any dimen- sion}

The bounds for 1-particles Hamiltonians, sufficient for our purposes, are established in a number of papers on the conventional Fractional Moment Method; cf. [1, 2, 3]. The scaling procedure in Section 4 allows one to establish the exponential decay bounds on the fractional moments of the $N$-particle Green functions. Section 3.2 provides then the derivation of the exponential decay bounds on the $\mathrm{N}$-particle eigenfunction correlators, thus completing the logical cycle $E F C(N-1) \rightarrow G F(N) \rightarrow E F C(N)$, i.e., the induction step $N-1 \rightsquigarrow N$ for the EFCs.

\subsection{Weakly disordered one-dimensional sys- tems}

Apart from the strongly disordered Anderson models in any dimension, Aizenman and Warzel [5] considered also the situation where the non-interacting system (formally speaking, in any dimension) features strong localization properties, and proved stability of Anderson localization under weak perturbations, i.e., with an interaction (of finite range) of sufficiently small amplitude; the latter may depend upon quantitative characteristics of localization in the non-interactive system.

The most appealing case is the $N$-particle system in $\mathbb{Z}^{1}$, for Anderson localization in one dimension is non-perturbative, i.e., occurs for arbitrarily small but nonzero disorder amplitude; cf. [25]. In fact, it occurs for any nontrivial probability distribution of the IID random potential, but the Fractional Moment Method can only be applied under a much more restrictive assumption of Hölder continuity of some or$\operatorname{der} \beta \in(0,1]$.

Below we consider only the extension to the onedimensional $N$-particle systems with exponentially decaying interaction, but it will be clear from the arguments that general result on stability under weak interactions remains valid in any dimension.

Recall that in order to carry out the inductive procedure and, in particular, to apply Lemma A.1, it suffices to find $m>0$ and $L_{0} \in \mathbb{N}^{*}$ such that (cf. (4.18)-(4.20))

$$
\Upsilon\left(L_{0}\right)+\mathrm{e}^{-\frac{m}{3} L_{0}}<M_{s}^{-1 / 2},
$$

for some $s \in(0,1)$ and $M_{s}$ defined in (3.2). It follows from the results of the fractional moment analysis of the onedimensional lattice Anderson model that for any amplitude $|g| \neq 0$ of the random potential $(x, \omega) \mapsto g V(x ; \omega)$, the eigenfunction correlators for the single-particle Hamiltonian $H(\omega)$ decay exponentially fast, with rate $m_{1}(g)>0$. It is closely related to the upper Lyapunov exponent $\gamma(E)$ for the 1D lattice Schrödinger operator; its asymptotical behaviour for small $|g|$, is well-known; cf. [24, 13, 30, 14, 31, 32].

The bottom line is that for $h=0$, i.e., in absence of interaction, $\Upsilon\left(L_{0}\right) \leq \mathrm{e}^{-\mu L_{0}}$ for some $\mu>0$, thus both terms in the LHS of (5.1) can be made arbitrary small by choosing $L_{0}$ large enough, thus guaranteeing the validity of (5.1) (for $h=0$ ). Since for $L_{0}$ fixed, the EFCs are continuous functions of the parameters of the Hamiltonian, the strict inequality of the form (5.1) is preserved for all $h$ with $|h| \leq h_{\circ}$, provided $h_{\circ}>0$ is small enough. This provides the required starting point for the quadratic recursive inequality for the sequence $\beta_{k}$, studied in Appendix A.

\section{Tunneling from split configurations}

Here we establish a key ingredient of the multi-scale inductive procedure, Lemma 6.1, allowing one to extend the techniques from [5] to the interactions of infinite range. The main argument does not use scale induction carried out in Section 4, which is one of the reasons we prove Lemma 6.1 separately.

\section{Lemma 6.1. Suppose that}

$$
\min \left[\mathrm{d}_{\mathcal{H}}(\mathbf{x}, \mathbf{y}), \operatorname{diam}(\mathbf{x}) \vee \operatorname{diam}(\mathbf{y})\right] \geq R>0 .
$$

Assume also that the n-particle EF correlators feature an exponential decay, with a positive decay exponent $m(=m(N-$ 1)). Then for some $c>0$, one has

$$
\mathbb{E}_{I}\left[|\mathbf{G}(\mathbf{x}, \mathbf{y})|^{s}\right] \leq A \mathrm{e}^{-c m R} .
$$

In other words, the EFC decay with exponent $m(N-1)$ in subsystems of up to $N-1$ particles results in exponential decay of the fractional moments of the $N$-particle Green functions, with exponent $m(N) \geq c m(N-1)$.

Proof. Without loss of generality, assume that diam $\mathbf{x} \geq$ $\max [\operatorname{diam} \mathbf{y}, R]$. Then for some partition of the index set $\{1, \ldots, N\}=\mathcal{J} \sqcup \mathcal{J}^{\mathrm{c}}$, we have $\mathbf{x}=\left(\mathbf{x}_{\mathcal{J}}, \mathbf{x}_{\mathcal{J}^{\mathrm{c}}}\right)$ with $\operatorname{dist}\left(\Pi \mathbf{x}_{\mathcal{J}}, \Pi \mathbf{x}_{\mathcal{J}^{c}}\right) \geq \ell(\mathbf{x}):=R /(N-1)$, and, respectively,

$$
\begin{aligned}
\mathbf{H} & =\mathbf{H}^{\mathcal{J}} \otimes \mathbf{1}+\mathbf{1} \otimes \mathbf{H}^{\mathcal{J}^{\mathrm{c}}}+\mathbf{U}^{\mathcal{J}, \mathcal{J}^{\mathrm{c}}} \\
& =\mathbf{H}^{\mathcal{J}, \mathcal{J}^{\mathrm{c}}}+\mathbf{U}^{\mathcal{J}, \mathcal{J}^{\mathrm{c}}} .
\end{aligned}
$$

We shall treat $\mathbf{U}^{\mathcal{J}, \mathcal{J}^{\mathrm{c}}}$ as a perturbation of $\mathbf{H}^{\mathcal{J}, \mathcal{J}^{\mathrm{c}}}$. By the second resolvent identity,

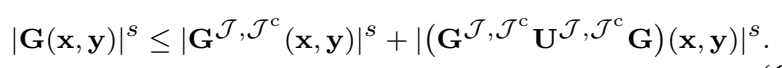

- Let us show first that

$$
\forall \mathbf{u}, \mathbf{v} \in \mathcal{Z}^{N} \quad \widehat{\mathbb{E}}^{I}\left[\left|\mathbf{G}^{\mathcal{J}, \mathcal{J}^{\mathrm{c}}}(\mathbf{u}, \mathbf{v})\right|^{2 s}\right] \leq A \mathrm{e}^{-m \mathrm{~d}_{\mathrm{S}}(\mathbf{u}, \mathbf{v})} .
$$

Indeed, by induction in the number of particles we know that

$$
\begin{aligned}
\widehat{\mathbb{E}}^{I}\left[Q^{\mathcal{J}}\left(\mathbf{x}_{\mathcal{J}}, \mathbf{y}_{\mathcal{J}} ; \mathbb{R}\right)\right] & \leq A \mathrm{e}^{-m \mathrm{~d}_{\mathcal{H}}\left(\mathbf{x}_{\mathcal{J}}, \mathbf{y}_{\mathcal{J}}\right)}, \\
\widehat{\mathbb{E}}^{I}\left[Q^{\mathcal{J}^{\mathrm{c}}}\left(\mathbf{x}_{\mathcal{J}^{\mathrm{c}}}, \mathbf{y}_{\mathcal{J}^{\mathrm{c}}} ; \mathbb{R}\right)\right] & \leq A \mathrm{e}^{-m \mathrm{~d}_{\mathcal{H}}\left(\mathbf{x}_{\mathcal{J}^{\mathrm{c}}}, \mathbf{y}_{\mathcal{J}^{\mathrm{c}}}\right)},
\end{aligned}
$$

and by (B.1), using the bounds $0 \leq Q^{\mathcal{J}}, Q^{\mathcal{J}^{\mathrm{c}}} \leq 1$,

$\widehat{\mathbb{E}}^{I}\left[Q^{\mathcal{J}} Q^{\mathcal{J}^{\mathrm{c}}}\right] \leq \mathrm{e}^{-m \min \left[\mathrm{d}_{\mathcal{H}}\left(\mathbf{x}_{\mathcal{J}}, \mathbf{y}_{\mathcal{J}}\right), \mathrm{d}_{\mathcal{H}}\left(\mathbf{x}_{\mathcal{J}^{\mathrm{c}}}, \mathbf{y}_{\mathcal{J}^{\mathrm{c}}}\right)\right]} \leq \mathrm{e}^{\left.-m \mathrm{~d}_{\mathcal{H}}(\mathbf{x}, \mathbf{y})\right)}$ 
It follows from Lemma 3.3 that

$$
\begin{aligned}
\widehat{\mathbb{E}}^{I}\left[\left|\mathbf{G}^{\mathcal{J}, \mathcal{J}^{\mathrm{c}}}(\mathbf{u}, \mathbf{v})\right|^{s}\right] & \leq \frac{C}{1-s} \widehat{\mathbb{E}}^{I}\left[\left|Q^{\mathcal{J}, \mathcal{J}^{\mathrm{c}}}(\mathbf{u}, \mathbf{v})\right|^{s}\right] \\
& \leq \frac{C}{1-s} A \mathrm{e}^{-m \mathrm{~d}_{\mathcal{H}}(\mathbf{u}, \mathbf{v})} .
\end{aligned}
$$

- Consider the perturbation term in (6.2). Setting

$$
\ell_{\mathcal{J}}(\mathbf{w}):=\mathrm{d}_{\mathcal{H}}\left(\mathbf{w}_{\mathcal{J}}, \mathbf{w}_{\mathcal{J}^{c}}\right)
$$

we have

$$
\begin{aligned}
\epsilon_{R} & \left.=\epsilon_{R}(\omega):=\mid \mathbf{G}^{\mathcal{J}, \mathcal{J}^{\mathrm{c}}} \mathbf{U G}\right)\left.(B x, \mathbf{y})\right|^{s} \\
& \leq \sum_{\ell_{\mathcal{J}}(\mathbf{w}) \leq R / 4} \widehat{\mathbb{E}}^{I}\left[\left|\mathbf{G}^{\mathcal{J}, \mathcal{J}^{\mathrm{c}}}(\mathbf{x}, \mathbf{w})\right|^{s}|\mathbf{U}(\mathbf{w})|^{s} \mathbf{G}\left(\mathbf{w},\left.\mathbf{y}\right|^{s}\right]\right. \\
& +\sum_{\ell_{\mathcal{J}}(\mathbf{w})>R / 4} \widehat{\mathbb{E}}^{I}\left[\left|\mathbf{G}^{\mathcal{J}, \mathcal{J}^{\mathrm{c}}}(\mathbf{x}, \mathbf{w})\right|^{s}|\mathbf{U}(\mathbf{w})|^{s} \mathbf{G}\left(\mathbf{w},\left.\mathbf{y}\right|^{s}\right]\right. \\
& \leq\|\mathbf{U}\| S_{1}+\mathrm{e}^{-a R s / 4} S_{2},
\end{aligned}
$$

where $\mathrm{e}^{-a s R / 4}$ is an upper bound on $\left|\mathbf{U}^{\mathcal{J}, \mathcal{J}^{\mathrm{c}}}\right|^{s}$ over the set $\left\{\mathbf{w}: \ell_{\mathcal{J}}(\mathbf{w})>R / 4\right\}$, and

$$
\begin{aligned}
& S_{1}=\sum_{\ell_{\mathcal{J}}(\mathbf{w}) \leq R / 4} \widehat{\mathbb{E}}^{I}\left[\left|\mathbf{G}^{\mathcal{J}, \mathcal{J}^{\mathrm{c}}}(\mathbf{x}, \mathbf{w})\right|^{s}|\mathbf{G}(\mathbf{w}, \mathbf{y})|^{s}\right], \\
& S_{2}=\sum_{\ell_{\mathcal{J}}(\mathbf{w})>R / 4} \widehat{\mathbb{E}}^{I}\left[\left|\mathbf{G}^{\mathcal{J}, \mathcal{J}^{\mathrm{c}}}(\mathbf{x}, \mathbf{w})\right|^{s}|\mathbf{G}(\mathbf{w}, \mathbf{y})|^{s}\right] .
\end{aligned}
$$

Using the Cauchy-Schwarz inequality and an a priori bound (3.1), we get

$$
\begin{aligned}
& S_{2} \leq \sum_{\ell_{\mathcal{J}}(\mathbf{w})>R / 4}\left(\widehat{\mathbb{E}}^{I}\left[\left|\mathbf{G}^{\mathcal{J}, \mathcal{J}^{\mathrm{c}}}(\mathbf{x}, \mathbf{w})\right|^{2 s}\right]\right)^{1 / 2} . \\
& \left(\widehat{\mathbb{E}}^{I}\left[|\mathbf{G}(\mathbf{w}, \mathbf{y})|^{2 s}\right]\right)^{1 / 2} \\
& \leq \sum_{\mathbf{w} \in \mathcal{Z}^{N}}\left(\widehat{\mathbb{E}}^{I}\left[\left|\mathbf{G}^{\mathcal{J}, \mathcal{J}^{\mathrm{c}}}(\mathbf{x}, \mathbf{w})\right|^{2 s}\right]\right)^{1 / 2} \cdot \frac{\text { Const }|g|^{-s}}{(1-2 s)^{1 / 2}},
\end{aligned}
$$

provided $2 s<1$, so the above expectations are finite. By (6.3) and Lemma B.2,

$$
\begin{aligned}
& \sum_{\mathbf{w} \in \mathcal{Z}^{N}} \widehat{\mathbb{E}}^{I}\left[\left|\mathbf{G}^{\mathcal{J}, \mathcal{J}^{\mathrm{c}}}(\mathbf{x}, \mathbf{w})\right|^{s}\right] \\
& \leq A \sum_{\mathbf{w} \in \mathcal{Z}^{N}} \mathrm{e}^{-m \mathrm{~d}_{\mathcal{H}}(\mathbf{x}, \mathbf{w})}=: A^{\prime}<+\infty
\end{aligned}
$$

thus

$$
\mathrm{e}^{-a s R / 4} S_{2} \leq C(s)|g|^{-s} \mathrm{e}^{-a s R / 4} .
$$

Next, assess $S_{1}$. Denote

$$
\mathrm{d}_{\mathcal{H}}^{\mathcal{J}}(\mathbf{x}, \mathbf{w}):=\max \left[\mathrm{d}_{\mathcal{H}}\left(\mathbf{x}_{\mathcal{J}}, \mathbf{w}_{\mathcal{J}}\right), \mathrm{d}_{\mathcal{H}}\left(\mathbf{x}_{\mathcal{J}^{\mathrm{c}}}, \mathbf{w}_{\mathcal{J}^{\mathrm{c}}}\right)\right]
$$

By Lemma B.1,

$$
\min _{\mathbf{w}: \ell \mathcal{J}(\mathbf{w}) \leq R / 4} \mathrm{~d}_{\mathcal{H}}^{\mathcal{J}}(\mathbf{x}, \mathbf{w}) \geq \frac{\ell(\mathbf{x})-R / 4}{2} \geq \frac{3 R}{8(N-1)}=: r_{0} .
$$

For any $r \geq r_{0}$, the number of configurations $\mathbf{w}$ with

$$
\mathrm{d}_{\mathcal{H}}^{\mathcal{J}}(\mathbf{x}, \mathbf{w})=r
$$

is bounded by $O\left(r^{N d}\right)$. Indeed, we have $R=a r_{0}$ with $a=$ $8(N-1) / 3$, so each $w_{i} \in \Pi \mathrm{w}$ must be inside the ball in $\mathcal{Z}$ centered at $x_{1}$ (or at any other particle position in $\mathbf{x}$ ) of radius

$$
r+\operatorname{diam} \mathbf{x}=r+R \leq c r, \quad c=1+\frac{8(N-1)}{R} .
$$

By assumption on the graph $\mathcal{Z}$, the cardinality of such ball is bounded by $C_{d}(c r)^{d}$, yielding the required bound.

$$
\begin{aligned}
& \sum_{\ell_{\mathcal{J}}(\mathbf{w}) \leq R^{\prime}} \widehat{\mathbb{E}}^{I}\left[\left|\mathbf{G}^{\mathcal{J}, \mathcal{J}^{\mathrm{c}}}(\mathbf{x}, \mathbf{w})\right|^{2 s}\right] \leq \sum_{r \geq R^{\prime}} C r^{N d} \mathrm{e}^{-m \mathrm{~d}_{\mathcal{H}}^{\mathcal{J}}(\mathbf{x}, \mathbf{w})} \\
& \leq C_{1} \mathrm{e}^{-c_{1}^{-1} m R}
\end{aligned}
$$

with $C_{1}, c_{1}^{-1}$ depending upon $N, d, m$ and uniformly bounded for all $m \geq 1, R \geq 1$ and $N \leq N^{*}$, with fixed $N^{*}$.

Taking into account (6.7), (6.6) and (6.11), we obtain

$$
\begin{gathered}
\widehat{\mathbb{E}}^{I}\left[\epsilon_{R}\right] \leq \frac{\text { Const } R}{|g|^{s}} \mathrm{e}^{-m R}+\frac{\text { Const }}{(1-2 s)|g|^{2 s}} \mathrm{e}^{-\frac{a s}{4} R} \\
\leq \frac{C(s)}{|g|^{s}} \mathrm{e}^{-\frac{a s}{4} R} .
\end{gathered}
$$

Collecting (6.3) and (6.12), the claim follows.

\section{Conclusion}

The Fractional Moments Method, since its inception in [1], has been successfully applied to various mathematical problems in the area of the disordered media, providing strong (exponential) bounds on the rate of decay of eigenfunction correlators. Its adaptation by Aizenman and Warzel [5] to the $N$-particle quantum systems with a nontrivial short-range inter-particle interaction was an important step towards understanding Anderson localization phenomena in realistic physical models with the number of particles proportional to the volume of the system in an (arbitrarily) large domain $\Lambda$ : $N=\rho|\Lambda|, \rho>0$. The latter have been recently studied by physicists (cf. [6, 23]). Recall that the existence of the socalled "metal-insulator transition" for single-particle models is accepted by the physics community as solidly established; the "insulator" regime corresponds to Anderson localization (with bound, square-summable quantum eigenstates), while the "metal" regime, which would probably manifest itself through the presence of a.c. spectrum and "extended states", remains a challenging problem in rigorous mathematical physics, except for specific models (almost periodic potentials in lattices $\mathbb{Z}^{d}$, or random potentials in the Cayley trees). In the own words of the authors of [6], "... the existence of the [many-body metal-insulator] transition is proven on the physical level of rigor".

Speaking of localization, the task of proving rigorously stability of Anderson localization in large disordered systems with positive density of particles is still awaiting mathematicians. The first steps in this direction (cf. [17], [5]) required a number of assumptions, including that of finite range of interaction, so the extension of the rigorous techniques of the MPMSA (cf. [17, 18]) and of the MPFMM (in the present paper, for discrete systems, and in [21], for continuous models) is the next logical step. It is comprehensible that heavy analytic techniques were required in [21] to treat unbounded, differential random Hamiltonians; in the present paper, we have shown that there is a simple, "soft" adaptation of the techniques, originally developed by Aizenman and Warzel in the framework of lattice $N$-particle systems, to the models with exponentially decaying interactions.

Finally, note that the latter class of interactions seems to be most relevant from the physical perspective, due to the wellknown phenomenon of Debye screening of the Coulomb interaction, resulting in exponential decay of electrostatic in- 
teraction at large distances between electrons in condensed matter.

\section{A Perturbed quadratic dynamics}

Lemma A.1. Let the sequence $\left\{L_{k}, k \geq 0\right\}$ satisfy the recursion $L_{k+1}=2 L_{k}+2$. Consider a sequence of positive numbers $\left\{\beta_{k}\right\}$ with $\beta_{0}<\mathrm{e}^{\nu}$, for some $\nu>0$, and satisfying

$$
\forall k \in \mathbb{N} \quad \beta_{k+1} \leq \frac{1}{2} \mathrm{e}^{-2 \nu} \beta_{k}^{2}+\frac{1}{2} \mathrm{e}^{-2 \nu} \mathrm{e}^{-2 \nu L_{k}} .
$$

Then for all $k \geq 1$

$$
\begin{aligned}
\beta_{k} & \leq \max \left\{\mathrm{e}^{-\nu L_{k}}, \mathrm{e}^{-\mu L_{k}}\right\}, \\
\mu & :=\frac{\nu+\ln \beta_{0}^{-1}}{1+\frac{L_{0}}{2}} .
\end{aligned}
$$

Proof. • First, note that if there exists $j \in \mathbb{N}$ such that

$$
\beta_{j} \leq \mathrm{e}^{-\nu L_{j}}
$$

then by induction, for all $k \geq j$ we have, using $L_{k}=\frac{1}{2} L_{k+1}-$ 1 ,

$$
\begin{aligned}
& \beta_{k+1} \leq \frac{1}{2} \mathrm{e}^{-2 \nu} \mathrm{e}^{-2 \nu L_{k}}+\frac{1}{2} \mathrm{e}^{-2 \nu} \mathrm{e}^{-2 \nu L_{k}} \\
& =\mathrm{e}^{-2 \nu} \mathrm{e}^{-2 \nu L_{k}}=\mathrm{e}^{-\nu L_{k+1}} .
\end{aligned}
$$

- Next, suppose that (A.4) never occurs, so for all $k$ we have

$$
\beta_{k+1}>\mathrm{e}^{-\nu L_{k}}
$$

Then $\frac{1}{2} \mathrm{e}^{-2 \nu} \beta_{k}^{2}+\frac{1}{2} \mathrm{e}^{-2 \nu} \mathrm{e}^{-\nu L_{k}}<\mathrm{e}^{-2 \nu} \beta_{k}^{2}$, and by (A.1),

$$
\forall k \geq 0 \quad \beta_{k+1}<\left(\mathrm{e}^{-\nu} \beta_{k}\right)^{2} .
$$

By induction, with $\mu$ given by (A.3) and $2^{k}=L_{k} /\left(L_{0}+2\right)$, for all $k \geq 0$,

$$
\beta_{k} \leq\left(\mathrm{e}^{-\nu} \beta_{0}\right)^{2^{k}}=\left(\mathrm{e}^{-\nu} \beta_{0}\right)^{\frac{L_{k}}{L_{0}+2}}=\mathrm{e}^{-\mu L_{k}},
$$

with $\mu=-\ln \left(a^{2} \beta_{0}^{2}\right) /\left(L_{0}+2\right)=\ln \left(a^{-1} \beta_{0}^{-1}\right) /\left(1+L_{0} / 2\right)$.

- Finally, if (A.6) holds on a finite integer interval [ $[0, j-1]]$, and then one has (A.4), the inequality (A.7) is still valid for $k \in[[0, j-1]]$, while the bounds (A.4), (A.5) take over for the remaining values $k \geq j$.

Consequently,

$$
\widehat{\Upsilon}_{k} \leq \mathrm{e}^{2 \nu} M_{s}^{1 / 2} \mathrm{e}^{-\widetilde{\mu} L_{k}}, \quad \widetilde{\mu}:=\min (\nu, \mu) .
$$

A reader familiar with the work by Germinet and Klein [22] can notice that the above proof is a variant an argument used in the proof of [22]*Theorem 5.1; cf. Eqns. (5.30)(5.32) in [22].

\section{B Some geometrical inequalities}

Note first that we have the following simple inequality for arbitrary configurations $\mathbf{x}, \mathbf{y} \in \mathcal{Z}^{N}$ and any partition $\left(\mathcal{J}, \mathcal{J}^{\mathrm{c}}\right)$ of the index set $\{1, \ldots, N\}$ :

$$
\min \left[\mathrm{d}_{\mathcal{H}}\left(\mathbf{x}_{\mathcal{J}}, \mathbf{y}_{\mathcal{J}}\right), \mathrm{d}_{\mathcal{H}}\left(\mathbf{x}_{\mathcal{J}^{c}}, \mathbf{y}_{\mathcal{J}^{c}}\right)\right] \geq \mathrm{d}_{\mathcal{H}}(\mathbf{x}, \mathbf{y}) .
$$

Indeed, assume w.l.o.g. that $\mathrm{d}_{\mathcal{H}}(\mathbf{x}, \mathbf{y})=\mathrm{d}\left(x_{\circ}, \Pi \mathbf{y}\right)$ for some $x_{\circ} \in \Pi \mathbf{x}$ (otherwise exchange $\mathbf{x} \leftrightarrow \mathbf{y}$ ). Fix any partition $\left(\mathcal{J}, \mathcal{J}^{\mathrm{c}}\right.$, then

$$
\begin{aligned}
\min _{x \in \Pi \mathbf{x}_{\mathcal{J}}} \mathrm{d}\left(x, \Pi \mathbf{y}_{\mathcal{J}}\right) & \geq \min _{x \in \Pi \mathbf{x}} \mathrm{d}(x, \Pi \mathbf{y}) \\
& =\mathrm{d}\left(x_{\circ}, \Pi \mathbf{y}\right)=\mathrm{d}_{\mathcal{H}}(\mathbf{x}, \mathbf{y}),
\end{aligned}
$$

thus $\mathrm{d}_{\mathcal{H}}\left(\mathbf{x}_{\mathcal{J}}, \mathbf{y}_{\mathcal{J}}\right) \geq \mathrm{d}_{\mathcal{H}}(\mathbf{x}, \mathbf{y})$. Similarly,

$$
\min _{x \in \Pi \mathbf{x}_{\mathcal{J}^{c}}} \mathrm{~d}\left(x, \Pi \mathbf{y}_{\mathcal{J}^{c}}\right) \geq \min _{x \in \Pi \mathbf{x}} \mathrm{d}(x, \Pi \mathbf{y})=\mathrm{d}_{\mathcal{H}}(\mathbf{x}, \mathbf{y}),
$$

which proves $\mathrm{d}_{\mathcal{H}}\left(\mathbf{x}_{\mathcal{J}^{\mathrm{c}}}, \mathbf{y}_{\mathcal{J}^{\mathrm{c}}}\right) \geq \mathrm{d}_{\mathcal{H}}(\mathbf{x}, \mathbf{y})$ and (B.1).

Further, one has

$$
\mathrm{d}_{\mathcal{H}}(\mathbf{x}, \mathbf{w}) \geq \boldsymbol{\rho}(\mathbf{x}, \mathbf{w})-\min [\operatorname{diam} \mathbf{x}, \operatorname{diam} \mathbf{w}] .
$$

For the proof, take $x_{c} i r c \in \Pi \mathbf{x}, w_{\circ} \in \Pi \mathbf{w}$ such that $R:=$ $\mathrm{d}\left(x_{\circ}, w_{\circ}\right)=\boldsymbol{\rho}(\mathbf{x}, \mathbf{w})$; then

$$
\begin{aligned}
\mathrm{d}_{\mathcal{H}}(\mathbf{x}, \mathbf{w}) & \geq \max _{x \in \Pi \mathbf{x}} \operatorname{dist}(x, \Pi \mathbf{w}) \geq \mathrm{d}\left(x_{\circ}, \Pi \mathbf{w}\right) \\
& \geq \mathrm{d}\left(x_{\circ}, w_{\circ}\right)-\operatorname{diam} \mathbf{w} \\
& =R-\operatorname{diam} \mathbf{w},
\end{aligned}
$$

and similarly $\mathrm{d}_{\mathcal{H}}(\mathbf{x}, \mathbf{w}) \geq R-\geq \operatorname{diam} \mathbf{w}$.

Lemma B.1. Given any configuration $\mathbf{u} \in \mathcal{Z}^{N}$ and an arbitrary partition of the index set $\{1, \ldots, N\}=\mathcal{J} \sqcup \mathcal{J}^{\mathrm{c}}$, denote

$$
\ell_{\mathcal{J}}(\mathbf{u})=\operatorname{dist}\left(\mathbf{u}_{\mathcal{J}}, \mathbf{u}_{\mathcal{J}^{\mathrm{c}}}\right)
$$

Then for all $\mathbf{x}, \mathbf{w} \in \mathcal{Z}^{N}$ the following inequality holds (cf. (6.8)):

$$
\mathrm{d}_{\mathcal{H}}^{\mathcal{J}}(\mathbf{x}, \mathbf{w}) \geq \frac{\ell_{\mathcal{J}}(\mathbf{x})-\ell_{\mathcal{J}}(\mathbf{w})}{2} .
$$

Proof. Let

$$
R:=\ell_{\mathcal{J}}(\mathbf{x}), \quad r:=\ell_{\mathcal{J}}(\mathbf{w}),
$$

so for some $j \in \mathcal{J}$ and $k \in \mathcal{J}^{\mathrm{c}}$ we have $\mathrm{d}\left(w_{j}, w_{k}\right)=r$. Recalling the definition of the distance $\mathrm{d}_{\mathcal{H}}^{\mathcal{J}}$ (cf. (6.8)),

$$
\mathrm{d}_{\mathcal{H}}^{\mathcal{J}}(\mathbf{x}, \mathbf{w}):=\max \left[\mathrm{d}_{\mathcal{H}}\left(\mathbf{x}_{\mathcal{J}}, \mathbf{w}_{\mathcal{J}}\right), \mathrm{d}_{\mathcal{H}}\left(\mathbf{x}_{\mathcal{J}^{\mathrm{c}}}, \mathbf{w}_{\mathcal{J}^{c}}\right)\right]
$$

we then have for some $j^{\prime} \in \mathcal{J}$ and $k^{\prime} \in \mathcal{J}^{\mathrm{c}}$

$$
\mathrm{d}\left(x_{j^{\prime}}, w_{j}\right) \leq \mathrm{d}_{\mathcal{H}}^{\mathcal{J}}(\mathbf{x}, \mathbf{w}), \quad \mathrm{d}\left(x_{k^{\prime}}, w_{k}\right) \leq \mathrm{d}_{\mathcal{H}}^{\mathcal{J}}(\mathbf{x}, \mathbf{w}),
$$

so by the triangle inequality for the graph-distance,

$$
\begin{aligned}
R \leq \mathrm{d}\left(x_{j^{\prime}}, x_{k^{\prime}}\right) & \leq \mathrm{d}\left(x_{j^{\prime}}, w_{j}\right)+\mathrm{d}\left(w_{j}, w_{k}\right)+\mathrm{d}\left(w_{k}, x_{k^{\prime}}\right) \\
& \leq r+2 \mathrm{~d}_{\mathcal{H}}^{\mathcal{J}}(\mathbf{x}, \mathbf{w}) .
\end{aligned}
$$

Therefore,

$$
\mathrm{d}_{\mathcal{H}}^{\mathcal{J}}(\mathbf{x}, \mathbf{w}) \geq \frac{R-r}{2}=\frac{\ell_{\mathcal{J}}(\mathbf{x})-\ell_{\mathcal{J}}(\mathbf{w})}{2} .
$$

Example. Let $\mathcal{Z}=\mathbb{Z}^{1}, N=2, \mathbf{x}=(-5,5), \mathbf{w}=(-1,1)$. Then for the unique nontrivial partition of $\{1,2\}$ into $\mathcal{J}=\{1\}$ and $\mathcal{J}^{\mathrm{c}}=\{2\}$, we have $\ell_{\mathcal{J}}(\mathbf{x})=10, \ell_{\mathcal{J}}(\mathbf{w})=2$, and

$$
\mathrm{d}_{\mathcal{H}}^{\mathcal{J}}(\mathbf{x}, \mathbf{w})=5-1=4=\frac{\ell_{\mathcal{J}}(\mathbf{x})-\ell_{\mathcal{J}}(\mathbf{w})}{2} .
$$

Lemma B.2.

$$
\sup _{\mathbf{x} \in \mathcal{Z}^{N}} \sum_{\mathbf{w} \in \mathcal{Z}^{N}} \mathrm{e}^{-m \mathrm{~d}_{\mathcal{H}}(\mathbf{x}, \mathbf{w})} \leq C(m, \mathcal{Z})<+\infty .
$$

Proof. Fix $r \geq 1$ and consider the set

$$
\mathcal{A}_{r}:=\left\{\mathbf{y}: \mathrm{d}_{\mathcal{H}}(\mathbf{x}, \mathbf{y})=r\right\} .
$$

Let $\mathbf{y} \in \mathcal{A}_{r}$, then

$$
\forall y \in \Pi \mathbf{y} y \in \bigcup_{j=1}^{n} \mathrm{~B}_{r}\left(x_{j}\right)
$$


thus

$$
\left|\mathcal{A}_{r}\right| \leq \prod_{j=1}^{n}\left|\cup_{j} \mathrm{~B}_{r}\left(x_{j}\right)\right| \leq C(N, d)\left(r^{d}\right)^{N} .
$$

Finally,

$$
\begin{gathered}
\sum_{\mathbf{w} \in \mathcal{Z}^{N}} \mathrm{e}^{-m \mathrm{~d}_{\mathcal{H}}(\mathbf{x}, \mathbf{w})} \leq \sum_{r \geq 1} \mathrm{e}^{-m r} C(N, d) r^{N d} \\
\leq C(m, N, d)<+\infty .
\end{gathered}
$$

\section{Boole's identity}

While there seems to be a consensus that the result stated below was first discovered and proved by George Boole in 1857, we hesitate to refer to the original work [7] as the source of the most comprehensive proof. Instead, we provide a very short (10 lines) and elementary proof given almost a century later by Lynn H. Loomis [28]. Boole's identity was rediscovered more than once and extended in various ways in the theory of the Hilbert transform, giving rise to a number of interesting applications.

Proposition C.1. Let be given real numbers $\lambda_{1}<\cdots<\lambda_{n}$ and positive real numbers $c_{1}, \ldots, c_{n}$. Then

$$
\forall t>0 \quad \operatorname{mes}\left\{x \in \mathbb{R}:\left|\sum_{i} \frac{c_{i}}{\lambda_{i}-x}\right|>t\right\}=\frac{2 \sum_{i} c_{i}}{t} .
$$

Proof. [Cf. [28]*Proof of Lemma 1]. We assess first the Lebesgue measure of the set $S_{+}$where $f(x):=\sum_{i} \frac{c_{i}}{x-\lambda_{i}}>t$. Since for all $x \notin\left\{\lambda_{1}, \ldots, \lambda_{n}\right\}$

$$
f^{\prime}(x)=\sum \frac{-c_{i}}{\left(\lambda_{i}-x\right)^{2}}<0,
$$

there are exactly $n$ roots $\kappa_{i}$ of the equation $f(x)=t$, and one has $\lambda_{i}<\kappa_{i}<\lambda_{i+1}, \kappa_{n}>\lambda_{n}$, thus $S_{+}=\sqcup_{i=1}^{n} I_{i}, I_{i}=\left(\lambda_{i}, \kappa_{i}\right)$, and mes $S_{+}=\sum_{i}\left(\kappa_{i}-\lambda_{i}\right)$.

Next, multiplying the equation $f(x):=\sum_{i} \frac{c_{i}}{\lambda_{i}-x}=t$ by $\prod_{i}\left(x-\lambda_{i}\right)$, we see that $\kappa_{i}$ are the roots of the polynomial admitting two equivalent representations

$$
t \prod_{i}\left(x-\lambda_{i}\right)-\sum_{i=1}^{n} c_{i} \prod_{j \neq i}\left(x-\lambda_{j}\right) \equiv t \prod_{i}\left(x-\kappa_{i}\right) .
$$

The identity for the sub-principal coefficients gives

$$
t \sum_{i} \lambda_{i}+\sum_{i} c_{i}=t \sum_{i} \kappa_{i}
$$

yielding mes $S_{+}=\sum_{i}\left(\kappa_{i}-\lambda_{i}\right)=t^{-1} \sum_{i} c_{i}$. Similarly, mes $\{x$ : $f(x)<-t\}=t^{-1} \sum_{i} c_{i}$.

\section{Proof of Lemma 3.1}

We focus on the case where $u_{1} \neq u_{2}$. The remaining case with $u_{1}=u_{2}$ is quite similar and even simpler; see the complete proof for $u_{1}=u_{2}$ in the manuscript [10].

As we shall see, the relevant representation of the random operator at hand is $g \mathbf{V}(\omega)+\mathbf{A}$, with the nonrandom component $\mathbf{A}=\mathbf{H}_{0}+\mathbf{U}$, and we work with the resolvent $\mathbf{G}_{g}(E)=(g \mathbf{V}(\omega)+\mathbf{A}-E)^{-1}$. The random field $V$ is assumed bounded, $\|V(x ; \cdot)\|_{\infty}<+\infty$, and it suffices to assume that $\|V(x ; \cdot)\|_{\infty} \leq 1$, for larger values are simply obtained by taking $|g|$ larger. In fact, even the particular model where $V \sim \operatorname{Unif}([0,1])$ is of great interest, and it is one of the most popular models of disorder in physics. Then we can extract the factor $g$ and note that, with $\mathbf{B}_{g}:=g^{-1} \mathbf{A}, \lambda=g^{-1} E$,

$$
\begin{aligned}
& \widehat{\mathbb{E}}\left[\left|\mathbf{G}_{g}(\mathbf{x}, \mathbf{y} ; E)\right|^{s} \mid \mathfrak{F}_{\neq u_{1}, u_{2}}\right] \\
& =|g|^{-s} \widehat{\mathbb{E}}\left[\left(\mathbf{1}_{\mathbf{y}},\left(\mathbf{V}(\omega)+\mathbf{B}_{g}-\lambda\right)^{-1} \mathbf{1}_{\mathbf{x}}\right)\right] .
\end{aligned}
$$

In the rest of the proof, we work with the resolvent of the operator $\mathbf{V}(\omega)+\mathbf{B}_{g}$, at a rescaled energy $\lambda$ (which is fixed in the proof, anyway).

- Reduced probability space. Now the r.v. $V(x ; \omega)$ vary inside $I=[0,1]$ and admit a bounded probability density $p_{V},\left\|p_{V}\right\|_{\infty}=\bar{p}<\infty$. The conditional distribution of $V$ given $\mathfrak{F}_{\neq u_{1}, u_{2}}$ gives rise to the reduced probability space $(A, \widetilde{\mathbb{P}})$, where $A=I^{2}, \widetilde{\mathbb{P}}$ is absolutely continuous with respect to the Lebesgue measure mes $I_{I} \otimes$ mes $_{I}$ on $A$, with density $\left(v_{1}, v_{2}\right) \mapsto \bar{p}\left(v_{1}, v_{2}\right)=p_{V}\left(v_{1}\right) p_{V}\left(v_{2}\right) \leq \bar{p}^{2}$. Then for any non-negative random variable $\widetilde{\zeta}$ on $(\Omega, \mathbb{P})$ and any $s \in(0,1)$, the conditional expectation of $\widetilde{\zeta}^{s}$ given $\mathfrak{F}_{\neq u_{1}, u_{2}}$ has the form (below we allow the expectation to be $+\infty$ )

$$
\begin{aligned}
0 & \leq \widehat{\mathbb{E}}\left[\widetilde{\zeta}^{s}(\omega) \mid \mathfrak{F}_{\neq u_{1}, u_{2}}\right] \\
& =\int_{I} d v_{1} \int_{I} d v_{2} p_{V}\left(v_{1}\right) p_{V}\left(v_{2}\right) \zeta^{s}\left(v_{1}, v_{2} ; \cdot\right) \\
& \leq \bar{p}^{2} \int_{A} d v_{1} d v_{2} \zeta^{s}\left(v_{1}, v_{2} ; \bullet\right)
\end{aligned}
$$

where $\zeta\left(v_{1}, v_{2} ; \bullet\right)$ is obtained from $\widetilde{\zeta}(\omega)$ by identifying $v_{j} \equiv$ $V\left(u_{j} ; \omega\right)$, with the remaining degrees of freedom fixed by conditioning (they are symbolically represented by $\bullet$ ). Now $\zeta$ can be considered as a random variable on the square $I^{2}$ with the normalized Lebesgue measure. For the rest of the argument, $\mathbb{P}\{\}$ and $\widehat{\mathbb{E}}[]$ refer to this new probability space. Let $F_{\zeta}(t)=\mathbb{P}\{\zeta \leq t\}$, then

$$
\begin{aligned}
\widehat{\mathbb{E}}\left[\zeta^{s}\right] & =\int_{A} d v_{1} d v_{2} \zeta^{s}\left(v_{1}, v_{2} ; \cdot\right)=\int_{0}^{\infty} t^{s} d F_{\zeta}(t) \\
& =s \int_{0}^{\infty} t^{s-1}\left(1-F_{\zeta}(t)\right) d t .
\end{aligned}
$$

We shall return to (D.2), once we obtain a suitable upper bound of the tail probability distribution function (below mes is the Lebesgue measure on $A$ )

$$
1-F_{\zeta}(t)=\operatorname{mes}\left\{\left(v_{1}, v_{2}\right) \in A: \zeta\left(v_{1}, v_{2}\right)>t\right\} .
$$

- The Birman-Schwinger relation. Introduce the sets

$$
\mathbf{S}_{j}=\left(\left(\left\{u_{j}\right\} \times \mathcal{Z}\right) \cup\left(\mathcal{Z} \times\left\{u_{j}\right\}\right) \cap \Lambda, \quad j=1,2,\right.
$$

the (multiplication) operators

$$
0 \neq \mathbf{C}=\mathbf{1}_{\mathbf{S}_{1}}+\mathbf{1}_{\mathbf{S}_{2}} \geq 0, \quad \mathbf{D}=\mathbf{1}_{\mathbf{S}_{1}}-\mathbf{1}_{\mathbf{S}_{2}}
$$

and the random variables

$$
\xi=\frac{1}{2}\left(V\left(x_{1} ; \omega\right)+V\left(x_{2} ; \omega\right)\right), \quad \eta=\frac{1}{2}\left(V\left(x_{1} ; \omega\right)-V\left(x_{2} ; \omega\right)\right) .
$$

Then

$$
V\left(u_{1} ; \omega\right) \mathbf{1}_{\mathbf{S}_{1}}+V\left(u_{2} ; \omega\right) \mathbf{1}_{\mathbf{S}_{2}}=\xi \mathbf{C}+\eta \mathbf{D}
$$

and

$$
\begin{aligned}
\mathbf{H}(\omega) & =\widetilde{\mathbf{K}}(\omega)+g V\left(u_{1} ; \omega\right) \mathbf{1}_{\mathbf{S}_{1}}+g V\left(u_{2} ; \omega\right) \mathbf{1}_{\mathbf{S}_{2}}, \\
& =\widetilde{\mathbf{K}}+g \xi \mathbf{C}+g \eta \mathbf{D}=\mathbf{K}+g \xi \mathbf{C},
\end{aligned}
$$

where $\widetilde{\mathbf{K}}(\omega)$ is $\mathfrak{F}_{\neq u_{1}, u_{2}}$-measurable and $\mathbf{K}(\omega)=\widetilde{\mathbf{K}}(\omega)+\eta(\omega) \mathbf{D}$. 
The operator $\mathbf{C}$ is non-negative and not identically zero, so we can use the Birman-Schwinger identity for $\mathbf{K}_{E}=\mathbf{K}-E$ :

$$
\mathbf{C}^{1 / 2}\left(\mathbf{K}_{E}+g \xi \mathbf{C}\right)^{-1} \mathbf{C}^{1 / 2}=\left(\mathbf{C}^{1 / 2} \mathbf{K}_{E, \mathbf{C}}^{-1} \mathbf{C}^{1 / 2}+g \xi \mathbf{1}\right)^{-1},
$$

where the operator

$$
\mathbf{K}_{E, \mathbf{C}}:=\mathbf{C}^{1 / 2}\left(\mathbf{K}_{E}+g \xi \mathbf{C}\right)^{-1} \mathbf{C}^{1 / 2}
$$

is considered acting in the subspace of $\mathcal{H}$,

$$
\begin{aligned}
\mathcal{H}_{\left\{u_{1}, u_{2}\right\}} & =(\operatorname{Ker} \mathbf{C})^{\perp} \\
& =\operatorname{Span}\left\{\mathbf{1}_{\mathbf{w}}: \mathbf{w} \in \Lambda^{2}, \Pi \mathbf{w} \cap\left\{u_{1}, u_{2}\right\} \neq \varnothing\right\},
\end{aligned}
$$

containing in particular $\mathbf{1}_{\mathbf{x}}$ and $\mathbf{1}_{\mathbf{y}}$. Its relevance is explained by the fact that both $1_{x}$ and $1_{y}$ are eigenvectors of $C$ with positive eigenvalues,

$$
\mathbf{C} \mathbf{1}_{\mathbf{X}}=\mathbf{1}_{\mathbf{S}_{1}} \mathbf{1}_{\mathbf{X}}+\mathbf{1}_{\mathbf{S}_{2}} \mathbf{1}_{\mathbf{X}}=\left(\mathbf{N}_{u_{1}}(\mathbf{x})+\mathbf{N}_{u_{2}}(\mathbf{x})\right) \mathbf{1}_{\mathbf{X}}=\alpha_{\mathbf{X}} \mathbf{1}_{\mathbf{X}},
$$

with

$$
\mathbf{N}_{w}(\mathbf{u}):=\operatorname{card}\left\{j \in\{1,2\}: u_{j}=w\right\}
$$

(the number of particles in $\mathbf{u}$ at the position $u$ ), hence

$$
\begin{aligned}
& \mathbf{C}^{1 / 2} \mathbf{1}_{\mathbf{x}}=\alpha_{\mathbf{x}}^{1 / 2} \mathbf{1}_{\mathbf{x}}, \\
& \mathbf{C}^{1 / 2} \mathbf{1}_{\mathbf{y}}=\alpha_{\mathbf{y}}^{1 / 2} \mathbf{1}_{\mathbf{y}},
\end{aligned}
$$

with $1 \leq \alpha_{\mathbf{x}}, \alpha_{\mathbf{y}} \leq 2$. Therefore, with $\alpha:=\left(\alpha_{\mathbf{x}} \alpha_{\mathbf{y}}\right)^{-1 / 2} \in$ $[1,1 / 2]$,

$$
\begin{aligned}
\mathbf{G}(\mathbf{x}, \mathbf{y} ; E) & =\left(\mathbf{1}_{\mathbf{y}},\left(\mathbf{K}_{E}+g \xi \mathbf{C}\right)^{-1} \mathbf{1}_{\mathbf{x}}\right) \\
& =\alpha\left(\mathbf{1}_{\mathbf{y}}, \mathbf{C}^{1 / 2}\left(\mathbf{K}_{E}+g \xi \mathbf{C}\right)^{-1} \mathbf{C}^{1 / 2} \mathbf{1}_{\mathbf{x}}\right) \\
& =\alpha\left(\mathbf{1}_{\mathbf{y}},\left(\mathbf{C}^{1 / 2} \mathbf{K}_{E, \mathbf{C}}^{-1} \mathbf{C}^{1 / 2}+g \xi \mathbf{1}\right)^{-1} \mathbf{1}_{\mathbf{x}}\right)
\end{aligned}
$$

Since $\alpha \leq 1$, one has an implication: for any $t>0$,

$|\mathbf{G}(\mathbf{x}, \mathbf{y} ; E)|>t \Longrightarrow\left|\left(\mathbf{1}_{\mathbf{y}},\left(\mathbf{C}^{1 / 2} \mathbf{K}_{E, \mathbf{C}} \mathbf{C}^{1 / 2}+g \xi \mathbf{1}\right)^{-1} \mathbf{1}_{\mathbf{x}}\right)\right|>t$,

where the RHS refers to the (finite-dimensional) space $\mathcal{H}_{\left\{u_{1}, u_{2}\right\}}$

- The tail tale and the Boole formula. Consider the linear change of variables $\Phi:\left(v_{1}, v_{2}\right) \mapsto(\xi, \eta)=\left(\left(v_{1}+v_{2}\right) / 2,\left(v_{1}-\right.\right.$ $\left.v_{2}\right) / 2$ ) with Jacobian $=2$. Note that with $\left(v_{1}, v_{2}\right) \in A$, $\xi$ varies in $[0,1]$ and $\eta$ in $[-1 / 2,1 / 2]$.

Let $A^{\prime}:=\Phi(A) \subset[0,1] \times[-1 / 2,1 / 2]$, then by the Fubini theorem,

$$
\begin{aligned}
\int_{\mathbb{R}^{2}} d v_{1} d v_{2} \mathbf{1}_{A} \mathbf{1}_{M_{t}} & =2 \int_{-1 / 2}^{1 / 2} d \eta \int_{\mathbb{R}} d \xi \mathbf{1}_{A^{\prime}}(\xi, \eta) \mathbf{1}_{M_{t}} \circ \Phi^{-1}(\xi, \eta) \\
& \leq 2 \int_{-1 / 2}^{1 / 2} d \eta \int_{\mathbb{R}} d \xi \mathbf{1}_{M_{t}} \circ \Phi^{-1}(\xi, \eta) \\
& \leq 2 \cdot \sup _{\eta \in \mathbb{R}} \operatorname{mes}\left(\mathcal{M}_{t}(\eta)\right),
\end{aligned}
$$

where

$$
\begin{aligned}
& \mathcal{M}_{t}(\eta) \\
& =\left\{\xi:\left|\left(\mathbf{1}_{\mathbf{y}},\left(\mathbf{C}^{1 / 2} \mathbf{K}_{E, \mathbf{C}, \eta}^{-1} \mathbf{C}^{1 / 2}+g \xi \mathbf{1}\right)^{-1} \mathbf{1}_{\mathbf{x}}\right)\right|>t\right\} .
\end{aligned}
$$

The function

$$
\begin{aligned}
\mathcal{R}: \xi \mapsto & \left(\mathbf{1}_{\mathbf{y}},\left(\mathbf{C}^{1 / 2} \mathbf{K}_{E, \mathbf{C}, \eta}^{-1} \mathbf{C}^{1 / 2}+g \xi \mathbf{1}\right)^{-1} \mathbf{1}_{\mathbf{x}}\right) \\
& \equiv g^{-1}\left(\mathbf{1}_{\mathbf{y}},\left(g^{-1} \mathbf{C}^{1 / 2} \mathbf{K}_{E, \mathbf{C}, \eta}^{-1} \mathbf{C}^{1 / 2}+\xi \mathbf{1}\right)^{-1} \mathbf{1}_{\mathbf{x}}\right)
\end{aligned}
$$

is rational, with real simple poles,

$$
\mathcal{R}(\xi)=\sum_{j} \frac{g^{-1} c_{j}}{\lambda_{j}-\xi}, \quad \sum_{j}\left|g^{-1} c_{j}\right| \leq|g|^{-1}
$$

(by Bessel's inequality), so we can again apply the Boole formula,

$$
\operatorname{mes}\{\xi:|\mathcal{R}(\xi)|>t\}=\frac{2 \sum_{i} c_{i}}{t} \leq \frac{2}{g t} .
$$

Therefore,

$$
1=\operatorname{mes} A \geq \int_{\mathbb{R}^{2}} d v_{1} d v_{2} \mathbf{1}_{A} \mathbf{1}_{M_{t}} \leq 2 \cdot \frac{2}{g t}=\frac{4}{g t},
$$

yielding for the tail probability distribution function

$$
1-F_{\zeta}(t) \leq \min \left[1,4 g^{-1} t^{-1}\right] .
$$

- Calculation of the fractional moment. Return to the fractional moment in (D.2):

$$
\begin{aligned}
\widehat{\mathbb{E}}\left[\zeta^{s}\right] & =s \int_{0}^{\infty} t^{s-1}\left(1-F_{\zeta}(t)\right) d t \\
& \leq s \int_{0}^{\infty} t^{s-1} \min \left(1,4 g^{-1} t^{-1}\right) d t \\
& =s \int_{0}^{4 / g} t^{s-1} d t+s \int_{4 / g}^{+\infty} t^{s-2} d t \\
& =\frac{4^{s}}{g^{s}(1-s)} .
\end{aligned}
$$

Finally, for the conditional fractional moment in (3.1), we obtain the claim:

$$
\widehat{\mathbb{E}}\left[|\mathbf{G}(\mathbf{x}, \mathbf{y} ; E)|^{s} \mid \mathfrak{F}_{\neq u_{1}, u_{2}}\right] \leq \bar{p}^{2} \widehat{\mathbb{E}}\left[\zeta^{s}\right] \leq \frac{C}{|g|^{s}(1-s)} .
$$

\section{REFERENCES}

1. Aizenman, M., Molchanov, S. A., Localization at large disorder and at extreme energies: An elementary derivation. Commun. Math. Phys., 157, 245-278 (1993)

2. Aizenman, M., Localization at weak disorder: some elementary bounds. Rev. Math. Phys., 6, 1163-1182 (1994)

3. Aizenman, M., Schenker, J. H., Friedrich, R.M., Hundertmark, D., Finite-volume fractional-moment criteria for Anderson localization. Commun. Math. Phys., 224, 219-253 (2001)

4. Aizenman, M., Elgart, A., Naboko, S., Schenker, J. H., Stolz, G., Moment analysis for localization in random Schrödinger operators. Invent. Math., 163, 343-413, (2006)

5. Aizenman, M., Warzel, S., Localization bounds for multiparticle systems. Commun. Math. Phys., 290, 903-934 (2009)

6. Basko, D. M., Aleiner, I. L., Altshuler, B. L., Metal-insulator transition in a weakly interacting many-electron system with localized single-particle states. Ann. Phys., 321, 1126-1205 (2006)

7. Boole, G., On the comparison of transcendents, with certain applications to the theory of definite integrals. Philos. Trans. Royal Soc., 147, 780 (1857)

8. Birman, M. S., On the spectrum of singular boundary-value problems (in Russian). Mat. Sb. (N.S.), 55, 125-174 (1961) 
9. Chulaevsky, V., From fixed-energy localization analysis to dynamical localization: An elementary path. J. Stat. Phys., 154, 1391-1429 (2014)

10. Chulaevsky, V., Comprehensive proofs of localization in Anderson models with interaction. I. Two-particle localization estimates. Online available from: arXiv:math-ph/1410.1079 (2014)

11. Chulaevsky, V., Exponential decay of eigenfunctions in a continuous multi-particle Anderson model with sub-exponentially decaying interaction. Online available from: arXiv:math$\mathrm{ph} / 1408.4646$ (2014)

12. Chulaevsky, V., Boutet de Monvel, A., Suhov, Y., Dynamical localization for a multi-particle model with an alloy-type external random potential. Nonlinearity, 24, 1451-1472 (2011)

13. Campanino, M., Klein, A., Anomalies in the one-dimensional Anderson model. Commun. Math. Phys., 130, 441-456 (1990)

14. Chulaevsky, V., Spencer, T., Positive Lyapunov exponents for a class of deterministic potentials. Commun. Math. Phys., 168, 455-466 (1995)

15. Chulaevsky, V., Suhov, Y., Wegner bounds for a two-particle tight binding model. Commun. Math. Phys., 283, 479-489 (2008)

16. Chulaevsky, V., Suhov, Y., Eigenfunctions in a two-particle Anderson tight binding model. Commun. Math. Phys., 289, 701723 (2009)

17. Chulaevsky, V., Suhov, Y., Multi-particle Anderson localisation: induction on the number of particles. Math. Phys. Anal. Geom., 12, 117-139 (2009)

18. Chulaevsky, V., Suhov, Y., Multi-scale Analysis for Random Quantum Systems with Interaction.

19. Chulaevsky, V., Suhov, Y., Efficient Anderson localization bounds for large multi-particle systems. J. Spec. Theory (to appear)

20. Elgart, A, Tautenhahn, M., Veselić, I., Anderson localization for a class of models with a sign-indefinite single-site potential via fractional moment method. Ann. Henri Poincaré, 12, no. 8, 1571-1599 (2010)
21. Fauser, M., Warzel, S., Multiparticle localization for disordered systems on continuous space via the fractional moment method. Rev. Math. Phys., 27:4, 1550010 (2015)

22. Germinet, F., Klein, A., Bootstrap multi-scale analysis and localization in random media. Commun. Math. Physics, 222, 415-448 (2001)

23. Gornyi, I. V., Mirlin, A. D., Polyakov, D. G., Interacting electrons in disordered wires: Anderson localization and lowtemperature transport. Phys. Rev. Lett., 95, 206603 (2005)

24. Kappus, M., Wegner, F., Anomaly in the center of the band of the one-dimensional Anderson model. Z. Phys., B45, 15-21 (1981)

25. Kunz, H., Souillard, B., Sur le spectre des opérateurs aux différences finies aléatoires. Commun. Math. Phys., 78, 201246 (1980)

26. Klein, A., Nguyen, S. T., Bootstrap multiscale analysis for the multi-particle Anderson model. J. Stat. Phys., 151, no.5, 938973 (2013)

27. Klein, A., Nguyen, S. T., Bootstrap multiscale analysis and localization for multi-particle continuous Anderson Hamiltonians. Online available from: arXiv:math-ph/1311.4220 (2013)

28. Loomis, L. H., A note on the Hilbert transform. Bull. Amer. Math. Soc., 52, 1082-1086 (1946)

29. Martinelli, F., Scoppola, E., Remark on the absence of absolutely continuous spectrum for $d$-dimensional Schrödinger operators with random opotential for large disorder or low energy. Commun. Math. Phys., 97, 465-471 (1985)

30. Pastur, L., Figotin, L., Spectra of Random and Almost Periodic Operators. Birkhäuser: Berlin, New York, (1992)

31. Sadel, C., Schulz-Baldes, H., Lyapunov exponents at anomalies of $\mathrm{SL}(2, \mathbb{R})$-actions. Operator Theory: Adv. Appl., 174, 159-172 (2007)

32. Sadel, C., Schulz-Baldes, H., Scaling diagram for the localization length at a band edge. Ann. Henri Poincaré, 8, 1595-1621 (2007)

33. Schwinger, J., On the bound states of a given potential. Proc. Natl. Acad. Sci. USA, 47, 122-129 (1961) 